Conceptual study of an ICRH traveling-wave antenna system for low-coupling conditions as expected in DEMO

This content has been downloaded from IOPscience. Please scroll down to see the full text.

2016 Nucl. Fusion 56076009

(http://iopscience.iop.org/0029-5515/56/7/076009)

View the table of contents for this issue, or go to the journal homepage for more

Download details:

IP Address: 157.193.64.83

This content was downloaded on 08/05/2017 at 15:29

Please note that terms and conditions apply.

You may also be interested in:

Performance of the ITER ICRH system as expected from TOPICA and ANTITER II modelling

A. Messiaen, R. Koch, R.R. Weynants et al.

Recent advances in physics and technology of lon Cyclotron Resonance Heating in view of future fusion reactors

J Ongena, A Messiaen, Ye O Kazakov et al.

ICRH antenna coupling physics and optimum plasma edge density profile. Application to ITER

A Messiaen and R Weynants

Study of the ITER ICRH system

A. Messiaen, M. Vervier, P. Dumortier et al.

Recent developments in ICRF antenna modelling

P.U. Lamalle, A.M. Messiaen, P. Dumortier et al.

Preparing ITER ICRF: development and analysis of the load resilient matching systems based on antenna mock-up measurements

A. Messiaen, M. Vervier, P. Dumortier et al.

3D electromagnetic optimization of the front face of the ITER ICRF antenna

F. Louche, P. Dumortier, A. Messiaen et al.

Helicon wave coupling in KSTAR plasmas for off-axis current drive in high electron pressure plasmas S.J. Wang, H.H. Wi, H.J. Kim et al.

TOPICA: analysis and design of ICRF antennas

V. Lancellotti, D. Milanesio, R. Maggiora et al. 


\title{
Conceptual study of an ICRH traveling-wave antenna system for low-coupling conditions as expected in DEMO
}

\author{
R. Ragona ${ }^{1,2}$ and A. Messiaen ${ }^{1}$ \\ 1 Laboratory for Plasma Physics, Royal Military Academy, (LPP-ERM/KMS), BE-1000, Brussels, \\ Belgium \\ 2 Ghent University, 9000 Ghent, Belgium \\ E-mail: riccardo.ragona@rma.ac.be
}

Received 26 January 2016, revised 14 April 2016

Accepted for publication 13 May 2016

Published 21 June 2016

\begin{abstract}
For the central heating of a fusion reactor ion cyclotron radio frequency heating (ICRH) is the first choice method as it is able to couple RF power to the ions without density limit. The drawback of this heating method is the problem of excitation of the magneto-sonic wave through the plasma boundary layer from the antenna located along the wall, without exceeding its voltage standoff. The amount of coupling depends on the antenna excitation and the surface admittance at the antenna output due to the plasma profile. The paper deals with the optimization of the antenna excitation by the use of sections of traveling-wave antennas (TWAs) distributed all along the reactor wall between the blanket modules. They are mounted and fed in resonant ring system(s). First, the physics of the coupling of a strap array is studied by simple models and the coupling code ANTITER II. Then, after the study of the basic properties of a TWA section, its feeding problem is solved by hybrids driving them in resonant ring circuit(s). The complete modeling is obtained from the matrices of the TWA sections connected to one of the feeding hybrid(s). The solution is iterated with the coupling code to determine the loading for a reference low-coupling ITER plasma profile. The resulting wave pattern up to the plasma bulk is derived. The proposed system is totally load resilient and allows us to obtain a very selective exciting wave spectrum. A discussion of some practical implementation problems is added.
\end{abstract}

Keywords: ICRH, traveling-wave antenna, DEMO, coupling, load resilient

(Some figures may appear in colour only in the online journal)

\section{Introduction}

The ion cyclotron radio frequency heating (ICRH) and current drive (CD) is a well established technique of tokamak auxiliary heating. The main advantages of ICRH\&CD are its ability to achieve central power deposition at large plasma densities and to directly heat the plasma ions. Its technology is mature and of relatively low cost up to the MW level. This auxiliary heating system is then a valuable choice for the demonstration reactor DEMO and the future reactor machines.

The launching structure of an ICRH system has to provide the requested coupled power to the plasma with a selective wave-number spectrum at an affordable voltage and current level in the launching and feeding system. The feeding system must also be almost insensitive to slow or fast plasma-loading variations to avoid generator tripping. In this paper, we will review the basic principles based on which a multi-strap array system could be designed as an optimal launching structure in resonant ring(s) configuration for the expected low-coupling conditions of DEMO.

The coupling of ion cyclotron range of frequencies (ICRF) power from the antenna to the plasma components is a twostep process. In the first step, the RF power transfer is achieved by exciting the fast magneto-sonic wave in the plasma. Then, 


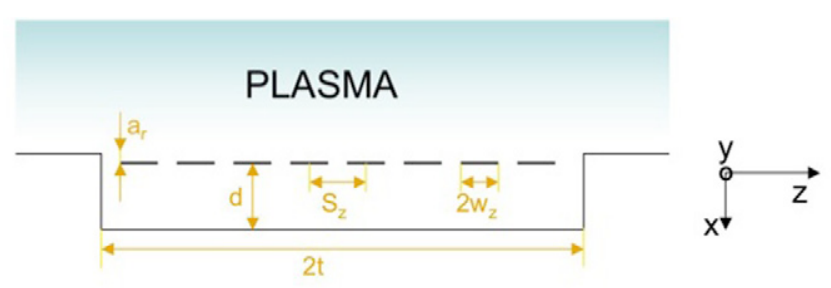

Figure 1. Toroidal cut of antenna box with strap array recessed in the wall. The geometrical parameters are indicated. The strap length along the $y$-axis is $l_{\text {str }}$. The geometrical parameters are indicated.

the wave moves to the chosen location in the plasma bulk where, in the second step, it is converted in kinetic energy of ions or electron particles. The first step is usually referred to as the coupling of the power to the plasma and the second as the absorption of the power in it. For this absorption, the two used main energy deposition mechanisms are: (i) heating of the minority ions and (ii) mode conversion. In the first scenario, the RF energy is transferred to a very low concentration of a minority species of ions (e.g. hydrogen minority in deuterium plasma) by means of an injected RF wave with frequency equal to the cyclotron frequency of the minority ion species. The majority species (one [1] of two components $[2,3])$ imposes the polarization of the magneto-sonic wave with a sufficient left-hand polarized component to accelerate the minority ions species. The second scenario uses the mode conversion through confluence of the fast wave with a slow wave such as the electrostatic ion Bernstein wave or ion cyclotron wave at the ion-ion hybrid layer in the plasma.

The radiating structure used to excite the magneto-sonic wave is usually embedded in the wall of the fusion device, in a recessed box. The antenna consists of an array of short (with respect to the vacuum wavelength) straps with a suitable current distribution in phase and amplitude that produces the desired wave number spectrum for the best coupling with good RF power absorption by the plasma species. The straps are aligned in the poloidal direction (or best perpendicular to the total steady magnetic field) in order to produce fields with the proper polarization to excite the fast wave into the plasma. This polarization corresponds to the transverse electric (TE/z with respect to the $z$-axis along the magnetic field) solution of the Maxwell's equations. The TE/z electromagnetic field excited by the antenna current distribution has to cross the plasma boundary region first to launch the fast magneto-sonic waves. The $\mathrm{TM} / z$ field component excited by the antenna is small with respect to the $\mathrm{TE} / z$ one because the considered strap currents are close to a current antinode (e.g. close to the strap short-circuited end with a strap length $\left.l_{\text {str }} \ll \lambda / 4\right)$. There is a non-negligible coupling between the $\mathrm{TM} / z$ and $\mathrm{TE} / z$ solutions inside the plasma profile if there is a confluence between the fast and slow modes as at the Alfven, lower hybrid or ionion Hybrid resonances. This situation at the plasma edge is not considered in this paper, but is analyzed in reference [4] with the estimated power flow to the slow wave computed by the code ANTITER II.

Let us consider the planar model of figure 1 showing a radiating strap array located in an antenna box recessed with respect to the vacuum vessel wall and in front of a plasma

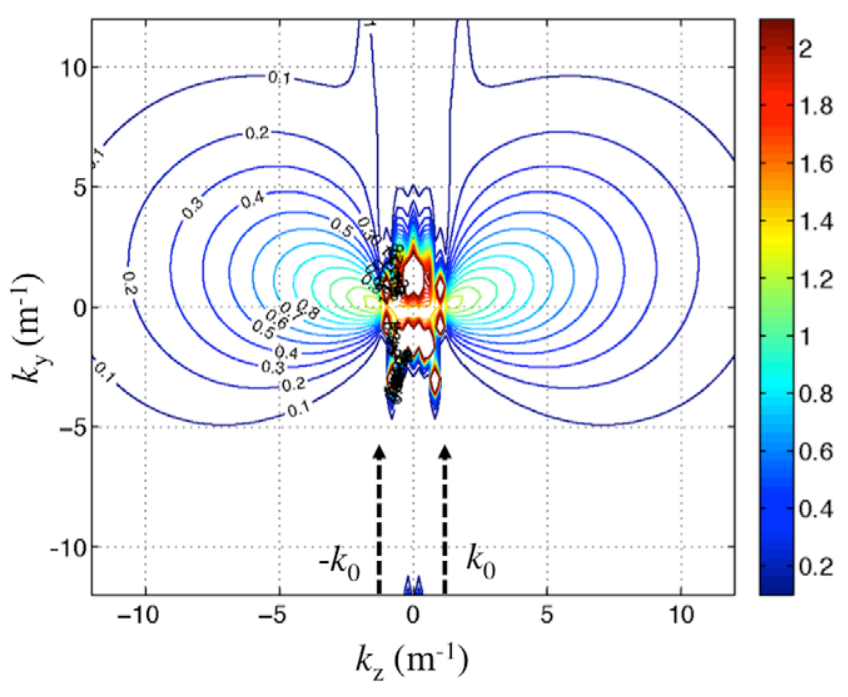

Figure 2. Contour of the normalized surface conductance $\operatorname{Re}\left(1 / \xi_{0}\right)$ in the $\left(k_{z}, k_{y}\right)$ plane at the antenna front due to plasma electron density profile of figure 3 (D-T plasma, $53 \mathrm{MHz}, B_{\text {sep }}=3.9 \mathrm{~T}$ ). The unit of the contour is $\mathrm{m}^{-1}$.

density profile. We take a Cartesian coordinate system with the $z$-axis along the magnetic field, the $x$-axis in the radial outwards direction and we make a Fourier analysis of the fields in their $\exp \left(\mathrm{i} k_{z} z+\mathrm{i} k_{y} y\right)$ components. Approximately, the $y$-axis represents the poloidal direction and the $z$-axis represents the toroidal direction. The total TE/ $z$ radiated power $P_{\text {rad }}$ can then be obtained from the Poynting theorem applied at the mouth of the antenna box in the $\left(k_{z}, k_{y}\right)$ space:

$$
\begin{aligned}
2 P_{\mathrm{rad}} & =\operatorname{Re}\left\{\left(1 /(2 \pi)^{2}\right) \iint E_{y} H_{z}{ }^{*} \mathrm{~d} k_{z} \mathrm{~d} k_{y}\right\} \\
& =\left\{1 /\left(4 \pi^{2} \omega \mu_{0}\right)\right\} \operatorname{Re}\left\{\iint\left|E_{y}\right|^{2} / \xi_{0}{ }^{*} \mathrm{~d} k_{z} \mathrm{~d} k_{y}\right\}
\end{aligned}
$$

where $\left|E_{y}\left(k_{z}, k_{y}\right)\right|^{2}$ is the spatial excitation function of the strap array at the antenna mouth, for the considered current distribution, and $\operatorname{Re}\left(1 / \xi_{0}\right)$ is the $\mathrm{TE} / z$ component of the normalized surface conductance of the external medium filled with inhomogeneous plasma $\left(\xi_{0}=E_{y} /\left(\omega B_{z}\right)\right.$ at the antenna box aperture). This surface conductance depends only on the characteristics of this medium. An example of its $\left(k_{z}, k_{y}\right)$ spectrum is given in figure 2 for the plasma electron density profile, assumed as the worst case for ITER (called '2010-low' [5]). This profile is considered as the one with the lowest electron density edge profiles in front of the antenna and, therefore, the worst for coupling. It is shown in figure 3 .

The surface conductance is obtained by numerical integration through this profile for a fine grid of $\left(k_{z}, k_{y}\right)$ values with as boundary condition the assumed total wave absorption in the plasma bulk (i.e. single-pass absorption). From the picture, some of the main characteristics of this function are highlighted: (i) the amplitude rapidly decreases with increasing $\left|k_{z}\right|$ or $\left|k_{y}\right|$ because the wave is evanescent when $k_{z}^{2}+k_{y}^{2}>k_{0}^{2}$ ( $k_{0}=\omega / c$ is the vacuum wave vector amplitude) from the antenna up to the cutoff density $N_{\mathrm{CO}}$ location in the plasma profile. $N_{\mathrm{CO}}$ depends on the frequency, magnetic field and ion species, but is also an increasing function of $\left|k_{z}\right|$ and $\left|k_{y}\right|$. 


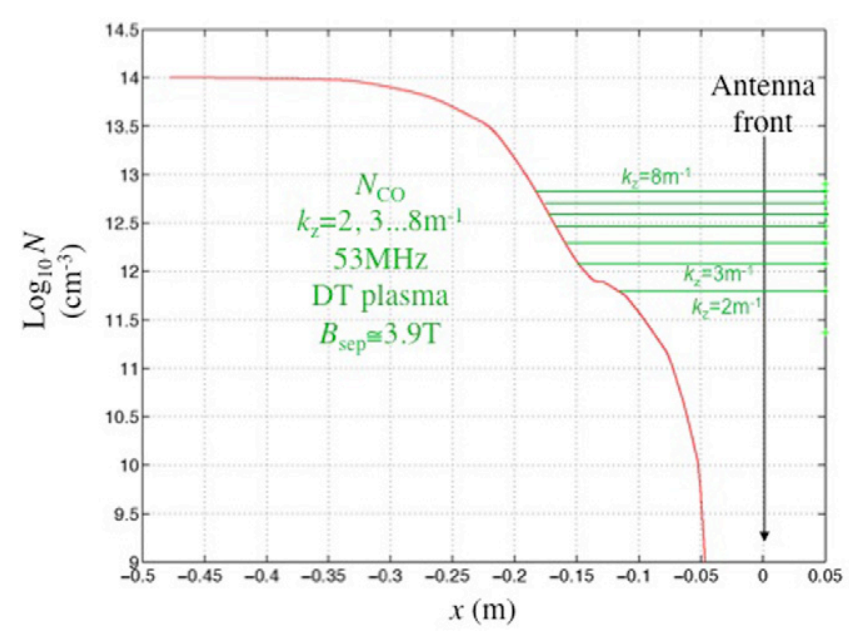

Figure 3. Reference ITER '2010-low' electron density profile with location of the fast magneto-sonic wave cut-off for different values of $k_{z}$. The reference antenna front position is indicated.

A detailed study of the effect of the plasma profile on the coupling is given in [6]. (ii) For $k_{z}^{2}+k_{y}^{2} \leqslant k_{0}^{2}$ the contribution of the coaxial modes clearly appears in figure 2 . These modes propagate between the wall and the plasma core [7] and lead to a deleterious RF power deposition at the plasma edge and the wall.

The electric field component $E_{y}\left(k_{z}, k_{y}\right)$ is the solution of the Maxwell's equations inside the box with the boundary condition at the box aperture expressed by its surface impedance $\xi_{0}\left(k_{z}, k_{y}\right)$ and is closely related to its excitation by the current distribution of the straps $J_{y}\left(k_{z}, k_{y}\right)$. As a consequence, the radiated power spectrum $P_{\text {rad }}\left(k_{z}, k_{y}\right)$ is also related to $\left|J_{y}\left(k_{z}, k_{y}\right)\right|$ but its amplitude quickly decreases when $\left|k_{z}\right|$ or $\left|k_{y}\right|$ increases due to the decrease of $\operatorname{Re}\left(1 / \xi_{0}\right)$. It could be inferred that the best portion of the spectrum for what concerns the coupling of power to the external medium, i.e. the plasma profile shown in figure 3 , is achieved in the region of the low $\left|k_{z}\right|$ and $\left|k_{y}\right|$ values exciding the region $k_{z}^{2}+k_{y}^{2} \leqslant k_{0}^{2}$ to avoid the coaxial modes if single-pass absorption can still be assumed.

The aim of antenna optimization is to maximize the excitation function $\left|E_{y}\left(k_{z}, k_{y}\right)\right|^{2}$ to obtain the maximum radiated power for a given surface admittance, i.e. a given plasma profile in front of the antenna.

In the next section, we will address this problem by (i) a simplified model and by (ii) a systematic comparison with the predictions of the antenna coupling code ANTITER II [8]. A more precise description of the antenna geometry can be obtained by finite elements modeling from the antenna code TOPICA [9]. This code includes a plasma description by the code FELICE [10]. The modeling of the ITER antenna array by TOPICA and ANTITER II is compared in [8]. The results of both codes are qualitatively and even quantitavely very similar. The advantages of the semi-analytical code ANTITER II is its fast computing time (typically a few minutes on a laptop depending on the requested integration precision and $\left(k_{y}, k_{z}\right)$ grid size) and a detailed physical insight into the results.

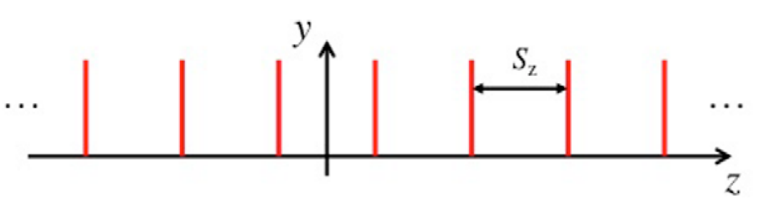

Figure 4. An infinite array of thin straps equally spaced with period $S_{z}$.

\section{Optimization of the antenna array}

The analysis of the antenna array will follow two steps. The first is based on a simplified model that takes into account two different cases: (a) an infinite array and (b) a finite array. The second step is based on the analysis by means of the ANTITER II code and will outline the main effects of the system on the coupling of power.

\subsection{Simplified model}

2.1.1. Case of an infinite array of thin straps (figure 4). The current amplitude $\left|I_{\mathrm{A}}\right|$ of each strap is the same and there is the same phase difference $\Delta \Phi$ between the currents of two consecutive straps. The corresponding surface current density distribution can be expressed by

$J_{y, \infty}(z)=\Sigma_{n}\left|I_{\mathrm{A}}\right| \exp (\mathrm{i} n \Delta \Phi) \delta\left(z-n S_{z}\right) \quad$ with $-\infty<n<\infty$.

The Fourier transform of this surface current distribution in the $k_{z}$ space is given by

$$
\begin{aligned}
J_{y, \infty}\left(k_{z}\right) & =2 \pi\left|I_{\mathrm{A}}\right| \sum_{p} \delta\left(k_{z} S_{z}-\Delta \Phi-p 2 \pi\right) \\
& =\left(2 \pi\left|I_{\mathrm{A}}\right| / S_{z}\right) \sum_{p} \delta\left(k_{z}-(\Delta \Phi-p 2 \pi) / S_{z}\right) \quad \text { with }-\infty<p<\infty .
\end{aligned}
$$

Therefore, the strap array selects discrete $k_{z}$ values, the first given by $k_{z 0}=\Delta \Phi / S_{z}$ and the others shifted by $p 2 \pi / S_{z}$ $(p= \pm 1, \pm 2, \pm 3 \ldots)$ with respect to the first one. The selected $k_{z}$ are closer when $S_{z}$ increases for a given $k_{z 0}$. The amplitude of the excitation of all selected $k_{z}$ values is equal and proportional to $\left|I_{\mathrm{A}}\right| / S_{z}$. If the strap width $2 w_{z}$ is finite, this spectrum is modulated by the function $\sin \left(k_{z} w_{z}\right) /\left(k_{z} w_{z}\right)$.

2.1.2. Case of a finite strap number equal to $n_{\text {str. }}$ The Fourier transform of this case is given by the convolution of the transforms of the infinite array given by (3) and the one of a function equal to 1 in the region from $z$ to $z+n_{\text {str }} S_{z}$ and equal to zero outside it. This yields the spectrum of a section of length $n_{\text {str }} S_{z}$ with $n_{\text {str }}$ straps equally spaced.

$$
\begin{aligned}
J_{y, n \text { str }} & =n_{\text {str }} I_{\mathrm{A}} \sum_{p} \sin \left(\xi_{p}\right) / \xi_{p} \quad \text { with } \\
\xi_{p} & =0.5 n_{\mathrm{str}} S_{z}\left\{k_{z}-(\Delta \Phi-p 2 \pi) / S_{z}\right\} .
\end{aligned}
$$

The delta functions of (3) in the $k_{z}$ spectrum are replaced by the $\sin \left(\xi_{p}\right) / \xi_{p}$ functions with main peaks at the same position, but of amplitude $n_{\text {str }}\left|I_{\mathrm{A}}\right|$. They become broader when the total length of the strap section $n_{\mathrm{str}} S_{z}$ decreases with their first zeros at distance $\Delta k_{z}= \pm 2 \pi /\left(n_{\text {str }} S_{z}\right)$ from the $k_{z \text {,peak }}=k_{z 0}+p 2 \pi / S_{z}$ values corresponding to the main peaks. The shape of the terms of (4) together with their dependence on $n_{\text {str }}$ and $S_{z}$ 

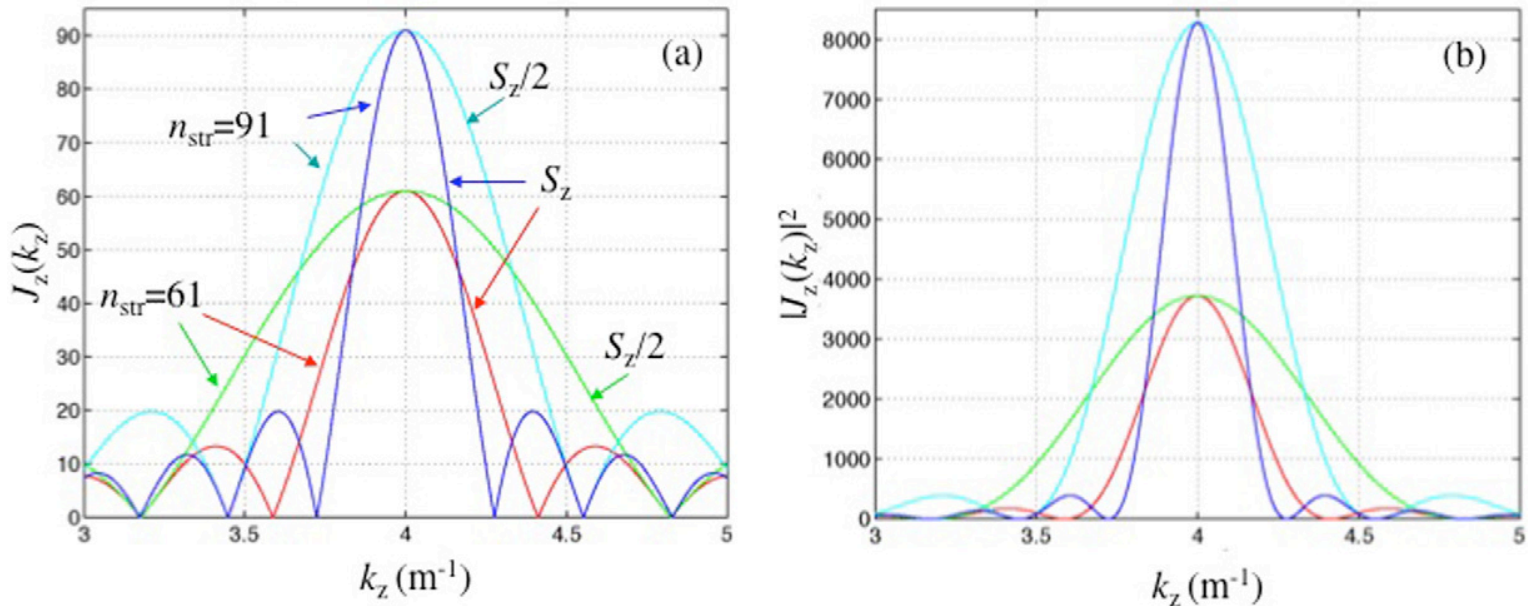

Figure 5. (a) $J_{z}\left(k_{z}\right)$ versus $k_{z}$ for an array with different finite strap number $n_{\text {str }}$ and inter-strap distance $\left(S_{z}=0.25 \mathrm{~m}\right)$ with $\Delta \Phi$ adjusted to obtain $k_{z 0}=4 \mathrm{~m}^{-1}$ and $(b)$ corresponding curves of $\left|J_{z}\left(k_{z}\right)\right|^{2}$ versus $k_{z}$.

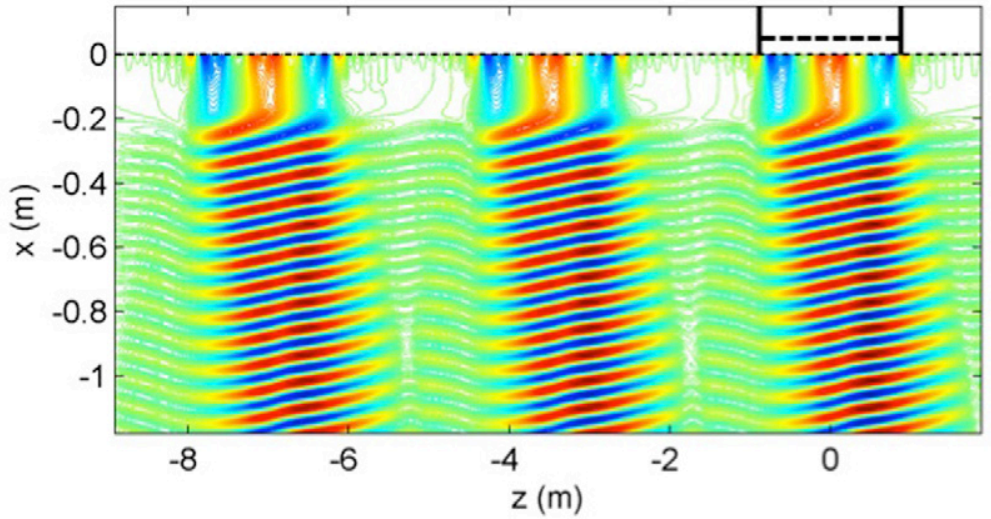

Figure 6. Contour plot of $\operatorname{Re}\left(B_{z}\right)$ at $t=0$ in the $(x, z)$ plane at $y=0$ starting from the antenna fronts in $x=0$ up to deep in the plasma profile of figure 3 . Three arrays, each with eight straps, are exciting the plasma. The arrays inter-strap phasing is adjusted to have $k_{z 0}=4 \mathrm{~m}^{-1}$ and the phasing of the three arrays is the same (D-T plasma, $53 \mathrm{MHz}$, separatrix at $x=-23 \mathrm{~cm}$ where $B_{\text {sep }}=2.9 \mathrm{~T}$ ). The strap position of one of the three arrays is shown in the figure.

is illustrated in figure $5(a)$. The integral of each term of (4) over $-\infty \leqslant k_{z} \leqslant \infty$ is equal to $2 \pi\left|I_{\mathrm{A}}\right| / S_{z}$ as for the case of the terms of (3) corresponding to $n_{\text {str }} \Rightarrow \infty$. Practically, due to the decrease of the surface conductance when $\left|k_{z}\right|$ increases (figure 2), only the first peak at $k_{z 0}=\Delta \Phi / S_{z}$ and its $p=-1$ (or $p=1$ ) side peak will have a significant contribution to $P_{\text {rad }}\left(k_{z}\right)$

The radiated power as expressed by (1) depends on the integral of $\left|E_{y}\right|^{2}$ multiplied by the surface conductance and, therefore, is expected to scale as the integral of $\left|J_{n s t r}\left(k_{z}\right)\right|^{2}$ over $k_{z}\left(-\infty \leqslant k_{z} \leqslant \infty\right)$ with respect to $n_{\text {str }}$ and $S_{z}$. Figure $5(b)$ shows the term of $\left|J_{n \text { str }}\left(k_{z}\right)\right|^{2}$ corresponding to figure $5(a)$. We have for each $p$ term of (4)

$$
\int\left|J_{n s t r}\left(k_{z}\right)\right|^{2} \mathrm{~d} k_{z}=2 \pi n_{\mathrm{str}} / S_{z} \text {. }
$$

In the next section, we will verify if the scaling of $P_{\text {rad }} \propto$ $n_{\text {str }} / S_{z}$ holds in the modeling of ANTITER II that fulfills all the boundary conditions of the coupling problem.

\subsection{Results of ANTITER II modeling}

ANTITER II considers the array geometry shown in figure 1 . It first computes the surface impedance at the antenna box aperture for deuterium-tritium (D-T) plasma with the electron density profile shown in figure 3 . This allows us to solve the Maxwell's equations with the appropriate boundary conditions in the antenna box and in the plasma profile [8]. The active radiated power is obtained from (1) and evaluated at the antenna front or equivalently from the EMF method applied on the surface of the straps. An example of the code output giving the contour plot of $\operatorname{Re}\left(\omega B_{z}\right)$ in front of an array of three sections of eight straps with $\Delta \Phi$ adjusted to obtain $k_{z 0}=\Delta \Phi / S_{z}=4 \mathrm{~m}^{-1}$ is given in figure 6 for the plasma profile of figure 3. A sketch of the antenna box of one of the travelingwave antennas (TWAs) section is also shown at its position. The figure displays the fields generated at very low plasma density in front of the antennas, crossing an evanescent region and reaching the density region where the fast magneto-sonic waves start to propagate and show their wave fronts. 

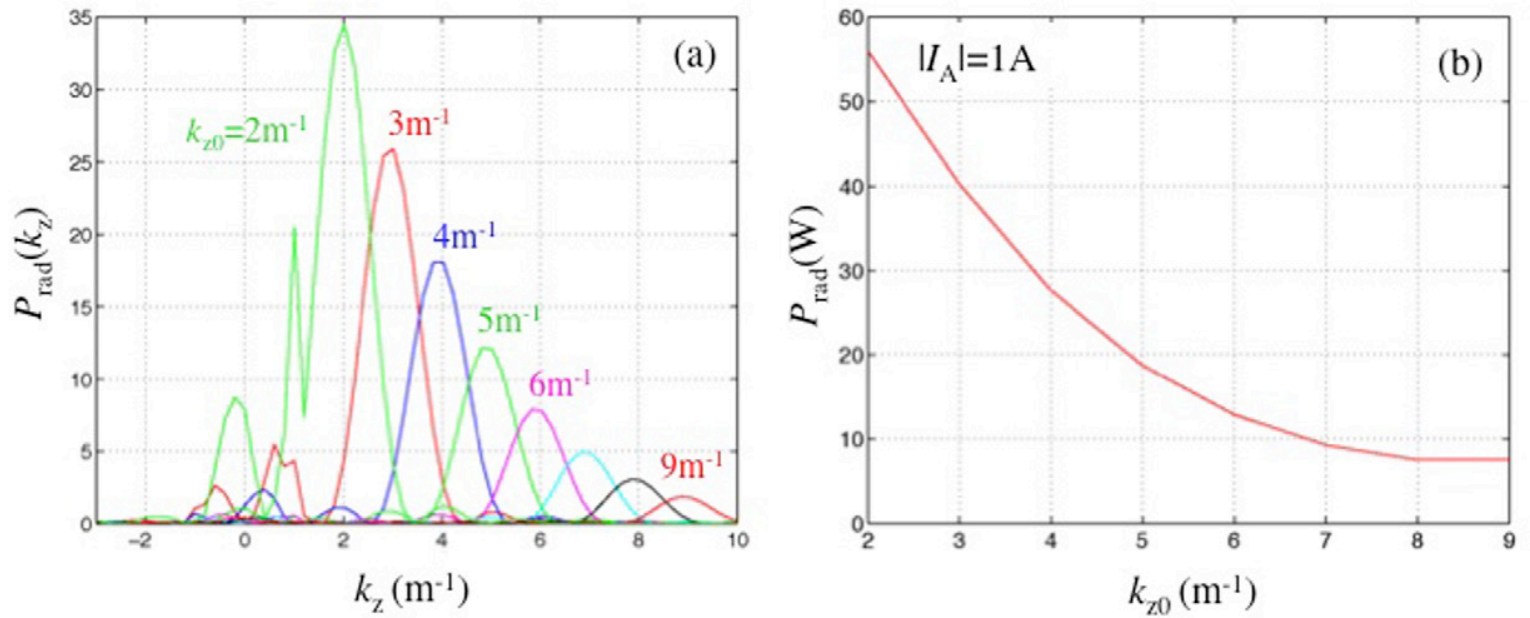

Figure 7. (a) Power $k_{z}$ spectrum with $\Delta \Phi$ adjusted to have $k_{z 0}=2,3, \ldots, 9 \mathrm{~m}^{-1}\left(S_{z}=0.37 \mathrm{~m}, n_{\mathrm{str}}=12, l_{\mathrm{str}}=0.915 \mathrm{~m}, 2 w_{z}=21 \mathrm{~cm}\right.$, $53 \mathrm{MHz}, B_{\text {sep }}=3.9 \mathrm{~T}$, D-T plasma with profile of figure 3), (b) corresponding evolution of total radiated power versus $k_{z 0}$ for $\left|I_{\mathrm{A}}\right|=1 \mathrm{~A}$ flowing in each strap.
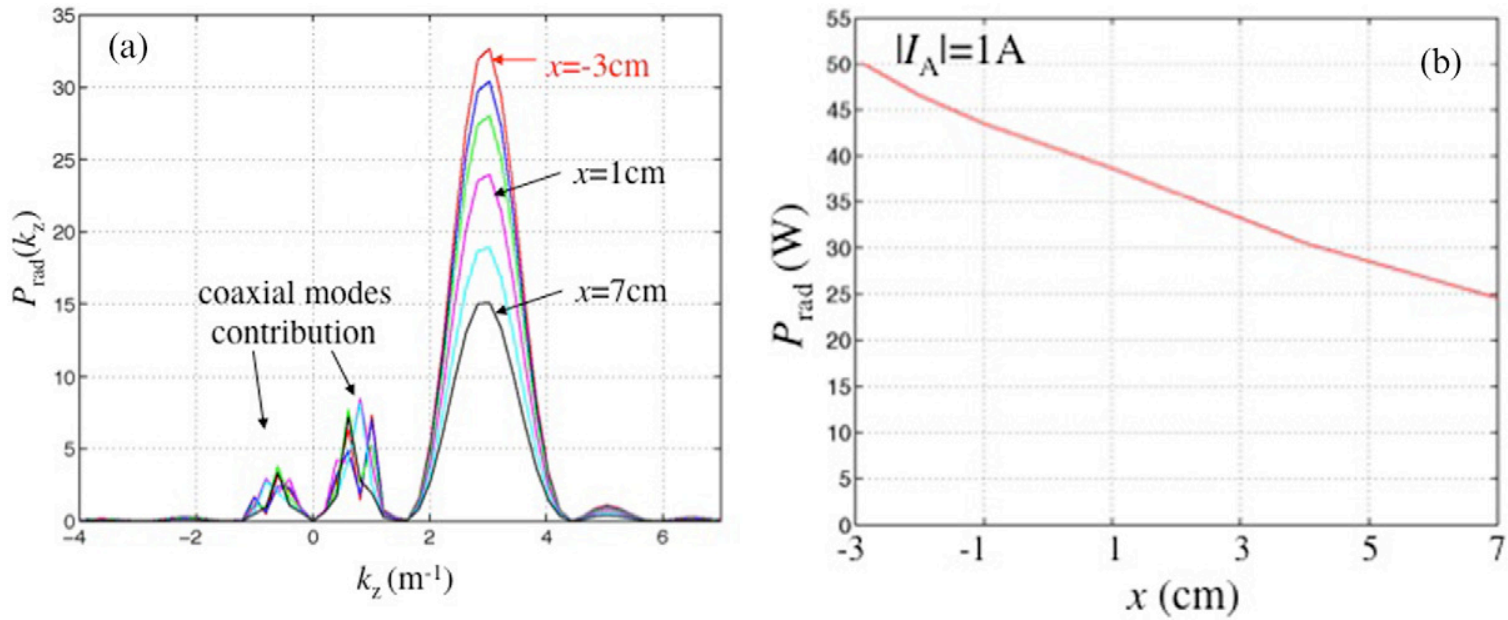

Figure 8. (a) Radiated power $k_{z}$ spectra for $\left|I_{\mathrm{A}}\right|=1 \mathrm{~A}$ in each strap and antenna front position $x=-3,-2,-1,1,4,7 \mathrm{~cm}$ in figure 3 . Here $k_{z 0}=3 \mathrm{~m}^{-1}$ is chosen. (b) Corresponding total radiated power versus antenna front position.

The main effects on the coupling first due to the surface conductance $\operatorname{Re}\left(1 / \xi_{0}\right)$ and second to the array geometry are summarized below.

2.2.1. Effect of the choice of $k_{z 0}=\Delta \Phi / S_{z}$. In figure 7(a) are shown the power $k_{z}$ spectra obtained with an array of 12 straps and the maximum radiation adjusted to obtain successively $k_{z 0}=2,3 \ldots 9 \mathrm{~m}^{-1}$. The amplitude of these selective spectra rapidly decreases when $k_{z 0}$ increases due to the decrease of the surface conductance with the larger distance of wave tunneling to the cutoff density as illustrated in figure 2 . One also observes for $\left|k_{z}\right|$ smaller or around $k_{0}=1.11 \mathrm{~m}^{-1}$ the contribution of the excitation at the peak side of the coaxial modes. It becomes large when $k_{z 0}$ is close to $k_{0}$ as for the case $k_{z 0}=2 \mathrm{~m}^{-1}$ of figure $7(a)$. The corresponding evolution of the total radiated power versus $k_{z 0}$ is given in figure $7(b)$ for $\left|I_{\mathrm{A}}\right|=1$ A flowing in each strap. The lack of a further decrease for $k_{z 0}=9 \mathrm{~m}^{-1}$ is due to the non-negligible excitation (even larger!) of the $p=-1$ peak occurring for $k_{z}=k_{z 0}-2 \pi / S_{z}=-7.9 \mathrm{~m}^{-1}$.
2.2.2. Effect of the distance of the antenna to the plasma profile. The sensitivity to this distance is illustrated in figure 8 . The $k_{z}$ power spectra of a 12 strap array are shown in figure $8(a)$ for different antenna front positions at $-3 \mathrm{~cm} \leqslant x \leqslant$ $7 \mathrm{~cm}$ (see figure 3). The reference one at $x=0 . \Delta \Phi$ is chosen to select a low $k_{z 0}=3 \mathrm{~m}^{-1}$ leading to a corresponding low cutoff density $N_{\mathrm{CO}}=11.710^{17} \mathrm{~m}^{-3}$ occurring at $x_{\mathrm{SOL}}=7.8 \mathrm{~cm}$ (see figure 3 ) in the considered D-T- $\left(\mathrm{He}^{3}\right)$ ion mix at $53 \mathrm{MHz}$ and low field site steady magnetic field $B_{\text {sep }} \cong 3.9$ T. Figure $8(b)$ indicates that the considered increase of distance $N_{\mathrm{CO}}$-antenna of $10 \mathrm{~cm}$ leads to a factor 2 of reduction of the array power capability for a given strap current amplitude.

2.2.3. Effect of the strap number $n_{\text {str }}$ in the array with constant $S_{z}$ and $k_{z 0}$. In this case, the antenna box length $2 L=n_{\text {str }} S_{z}$ increases proportionally to the number of straps. The results of a $4 \leqslant n_{\text {str }} \leqslant 16$ scan for $k_{z 0}=4 \mathrm{~m}^{-1}$ is shown in figure $9(a)$ for the power $k_{z}$ spectra and in figure $9(b)$ for the total radiated power. The selectivity increases with the strap number as expected from (4) and the total radiated 

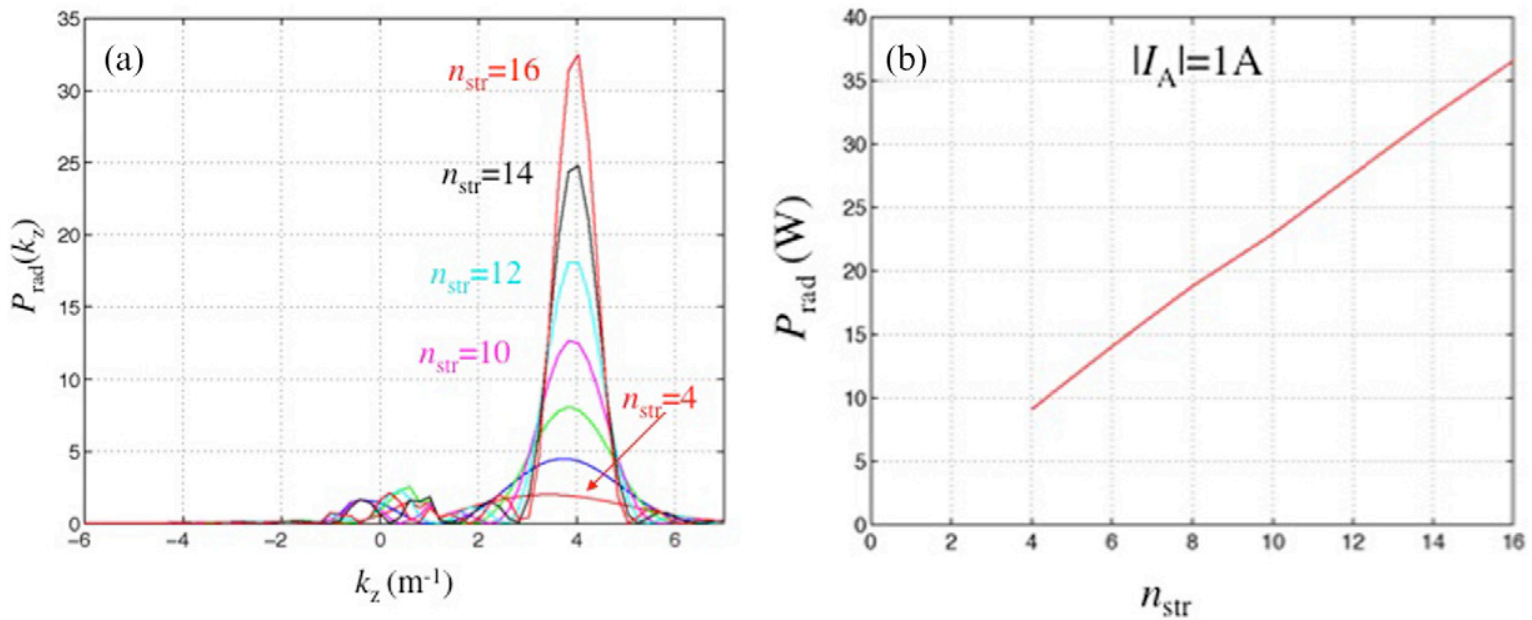

Figure 9. (a) Radiated power $k_{z}$ spectra for $\left|I_{\mathrm{A}}\right|=1 \mathrm{~A}$ in each strap versus the array strap number $n_{\text {str }}=4,6,8 \ldots 16$ with constant $S_{z}=0.37 \mathrm{~m}$ and parameters of figure 7 with $k_{z 0}=4 \mathrm{~m}^{-1}$. (b) Corresponding total radiated power versus strap number.
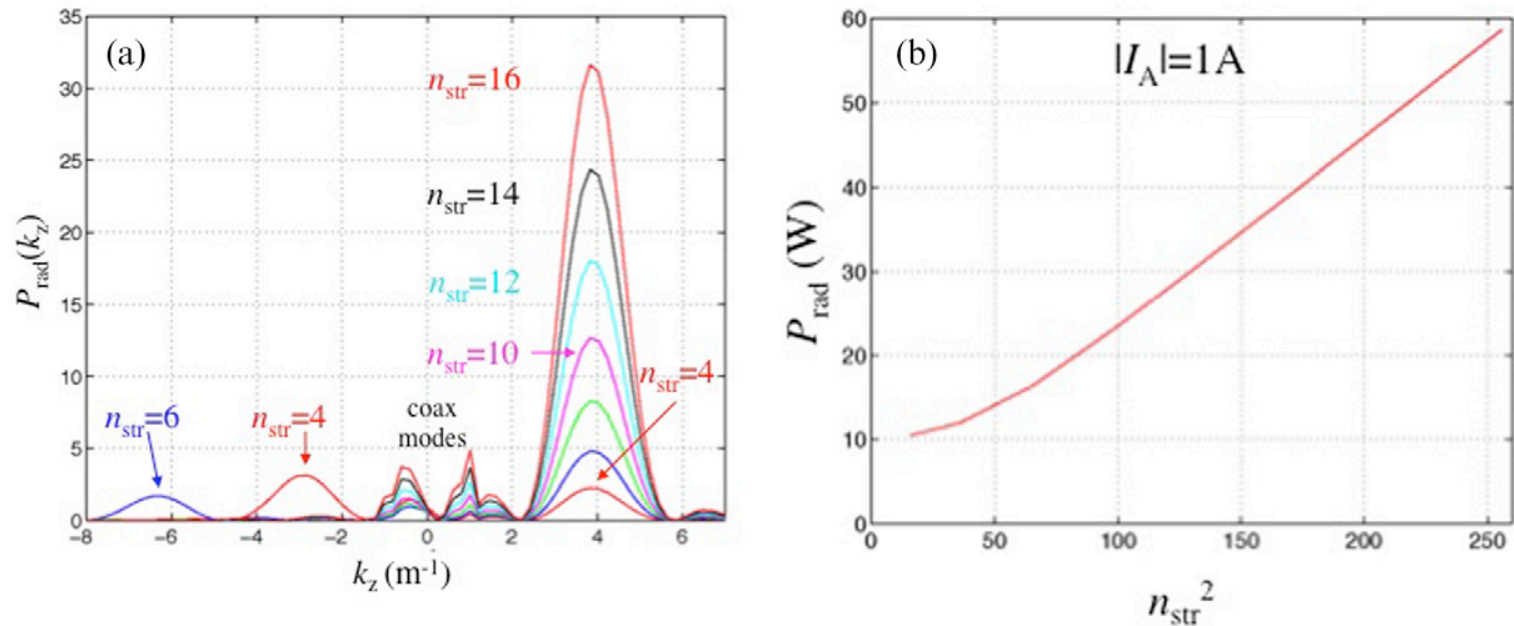

Figure 10. (a) Radiated power $k_{z}$ spectra for $\left|I_{\mathrm{A}}\right|=1 \mathrm{~A}$ in each strap versus the array strap number $n_{\text {str }}=4,6,8 \ldots 16$ with variable $S_{z}$, constant antenna box length and strap width (other parameters as for figure 7 with $k_{z 0}=4 \mathrm{~m}^{-1}$ ). (b) Corresponding total radiated power versus the square of the strap number.

power is proportional to the number of straps carrying the same current amplitude $\left|I_{\mathrm{A}}\right|$ in accordance with (5). The small excitation of coaxial modes for $\left|k_{z}\right| \leqslant k_{0}$ also appears in figure $9(a)$.

2.2.4. Effect of the strap number $n_{\text {str }}$ in the array with constant antenna box length $2 L$ and $k_{z 0}$. The number of straps is then increased by the reduction of their distance $S_{z}=2 L / n_{\text {str }}$ $(2 L=3.6 \mathrm{~m}) . S_{z}$ cannot be smaller than the strap width $2 w_{z}$ that we maintain constant and equal to the value of the preceding cases. Figure 10 $(a)$ shows the resulting power $k_{z}$ spectra for $4 \leqslant n_{\text {str }} \leqslant 16$. The contribution of the $p=-1$ side peak (at $k_{z}=k_{z 0}-2 \pi / S_{z}$ ) for the cases $n_{\mathrm{str}}=4$ and 5 with the largest $S_{z}$ clearly appears. Note also the coaxial mode contributions. Figure $10(b)$ gives the resulting value of the total radiated power $P_{\text {rad }}$ versus the square of the strap number. As expected from (5), $P_{\text {rad }}$ is proportional to $n_{\text {str }} / S_{z} \propto n_{\text {str }}^{2}$ in our case. The deviation at low $n_{\text {str }}$ is due to the non-negligible side-peak contribution.

\subsubsection{Effect of the strap dimensions.}

(a). The strap length $l_{\text {str. }}$ The influence of the strap length $l_{\text {str }}$ is large. The radiated power for a given strap current decreases strongly when $l_{\text {str }}$ becomes shorter as shown in the power $k_{z}$ spectra of figure $11(a)$. It decreases more than proportionally to $l_{\text {str. }}$. This clearly appears in figure $11(b)$ where the normalized ratio of $P_{\text {rad }}$ and $l_{\text {str }}$ is plotted as a function of $l_{\text {str }}$. This normalized ratio is the mean distributed radiation resistance $R_{\mathrm{A}}=2 P_{\mathrm{rad}} /\left(\left|I_{\mathrm{A}}\right| 2 n_{\mathrm{str}} l_{\mathrm{str}}\right)$ of the equivalent strip-line to the strap. On the other hand, the strap voltage needed to drive the strap current amplitude $\left|I_{\mathrm{A}}\right|$ is increasing with $l_{\text {str }}$ due to the strap inductance increase. The radiated power capability for a given strap voltage amplitude $|V|$ is expressed by the mean strap input conductance $G_{\text {str }}=2 P /|V|^{2}=\operatorname{Re}\left(1 / z_{\mathrm{a}}\right) \approx r_{\mathrm{A}} / x_{\mathrm{A}}^{2}$ (with $P=P_{\text {rad }} / n_{\text {str }}$ and $z_{\mathrm{A}}=r_{\mathrm{A}}+\mathrm{i} x_{\mathrm{A}}$ being the mean strap input impedance; note that $r_{\mathrm{A}}=R_{\mathrm{A}} l_{\text {str }}$ when $\left.l_{\text {str }} \ll \lambda / 4\right)$. This conductance decreases for larger $l_{\text {str }}$ because the increase of $r_{\mathrm{A}}$ is over-compensated by the one of $x_{\mathrm{A}}^{2}$. This is shown in figure $11(c)$. To benefit from the substantial $r_{\mathrm{A}}$ increase with 

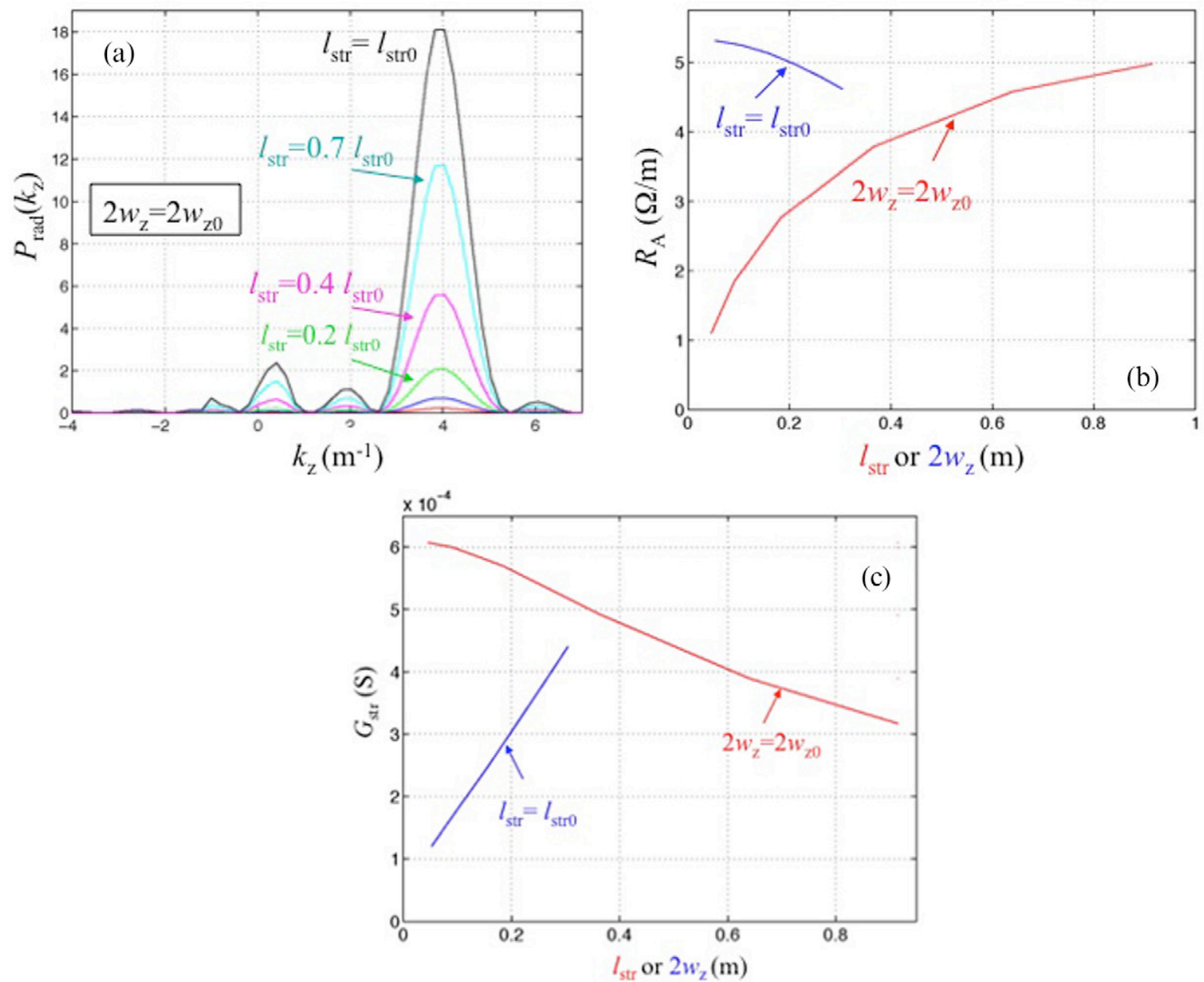

Figure 11. (a) Radiated power $k_{\mathrm{z}}$ spectra for $\left|I_{\mathrm{A}}\right|=1 \mathrm{~A}$ in each strap versus the array strap length $l_{\mathrm{str}}=(0.05,0.1,0.2,0.4,0.7,1) l_{\mathrm{str} 0}$ $\left(l_{\text {str } 0}=0.915 \mathrm{~m}\right.$, other parameters as for figure 7 with $\left.k_{z 0}=4 \mathrm{~m}^{-1}\right) .(b)$ Distributed mean strap resistance versus the strap length $l_{\text {str }}$ for $2 w_{z}=2 w_{z 0}=21 \mathrm{~cm}$ or versus strap width $2 w_{z}$ for $l_{\text {str }}=l_{\text {stro }}$. (c) Mean strap input conductance versus the strap length for $2 w_{z}=2 w_{z 0}=21 \mathrm{~cm}$ or versus strap width for $l_{\mathrm{str}}=l_{\mathrm{str} 0}$.

a longer strap without too large strap voltage increase, one can either (i) split the strap in sections with parallel feeding (as the strap triplets fed by a four-port junction in the ITER antenna design $[11,12]$ ) or (ii) use central grounding with push-pull feeding. These solutions also have the advantage of maintaining nearly constant the current amplitude all along the strap.

(b). The strap width $2 w_{z}$. We have already stated that the strap width modulates the amplitude of the peaks of $J_{y}\left(k_{z}\right)$ by the function $\sin \left(k_{z} w_{z}\right) /\left(k_{z} w_{z}\right)$ for a strap current density uniform in the $z$-direction. Its influence is small when we have $k_{z 0} w_{z} \ll$ 1. The resulting small decrease of $R_{\mathrm{A}}$ with larger strap width is shown in figure $11(b)$. On the other hand, an increase of this width leads to a strong reduction of the strap inductance and consequently a large increase of its mean conductance $G_{\text {str }}$ as shown in figure $11(c)$. Large straps are then beneficial for decreasing the voltage needed to drive the strap current, but are limiting the strap number per unit length of a TWA array.

2.2.6. Effect of the antenna box depth. The distance $d$ between the back plate of the antenna box and the strap (see figure 1) cannot be too small, because the image current induced by the back plate tends to cancel the strap radiation. This effect on the total radiated power $P_{\text {rad }}$ (for $\left|I_{\mathrm{A}}\right|=1 \mathrm{~A}$ flowing in each strap) and on the mean strap conductance $G_{\text {str }}$ is illustrated respectively in figures $12(a)$ and $(b)$. Note that an increase of $d$ from 5 to $40 \mathrm{~cm}$ leads to an increase of power capability of 7.7 times for a given strap current and of 1.46 times for a given strap voltage. The difference is due to the increase of the strap inductance with larger distance $d$.

2.2.7. Conclusions of the ANTITER study. From the present analysis, the radiated power of the array for a given strap current amplitude scales like $n_{\mathrm{str}} / S_{z}$ and is the largest for $k_{z 0}$ as low as possible, but sufficiently larger than $k_{0}$ to avoid significant coaxial mode excitation. This scaling requires array(s) with large $n_{\text {str }}$ and as small as possible inter-distance $S_{z}$. The voltage standoff is the limiting factor of $S_{z}$ : (i) the strap interdistance cannot be reduced too much and (ii) a reduction of the strap width $2 w_{z}$ leads to an increase of the strap inductance and, therefore, of the necessary voltage to drive the strap current.

The drawback of a large array is that it requires a complex feeding system with decoupling between the generators 

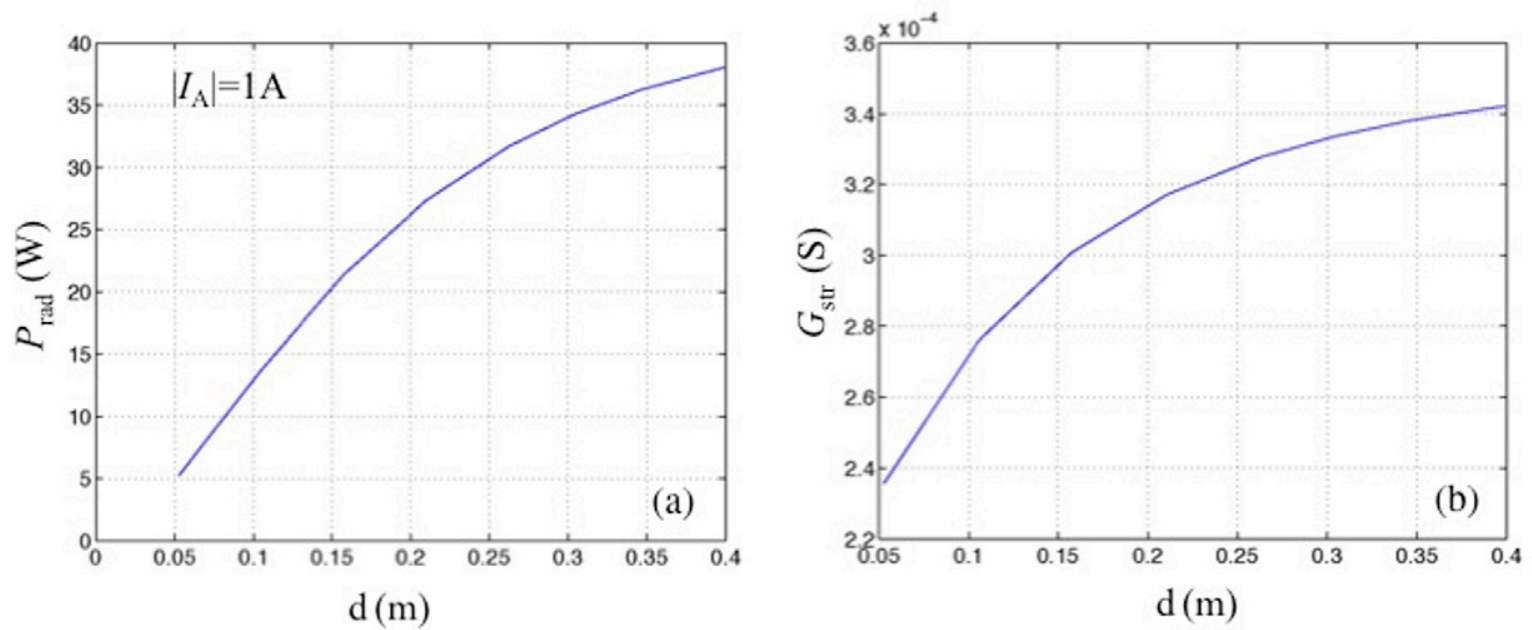

Figure 12. (a) Total radiated power versus the distance strap-back plate $d$ (see figure 1) for parameters of figure 7 with $k_{z 0}=4 \mathrm{~m}^{-1}$. (b) Corresponding mean strap input conductance versus $d$.

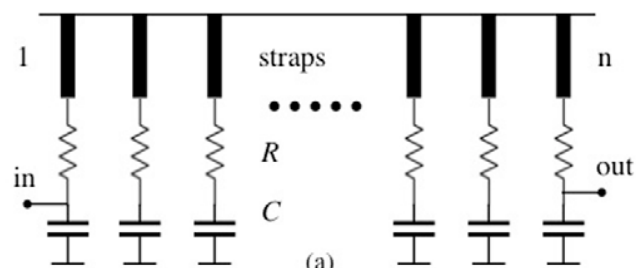

(a)

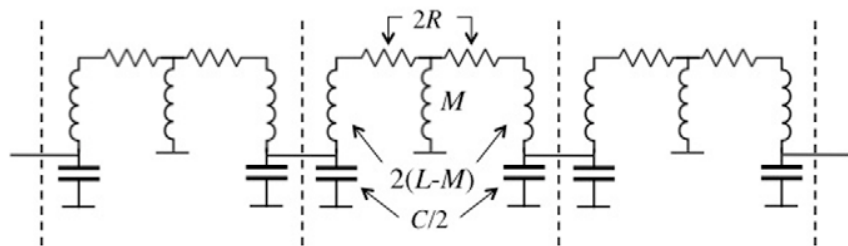

(b)

Figure 13. (a) Array of $n$ short-circuited straps connected to a radiation resistance $R$ and a tuning capacitor $C$. (b) Approximate equivalent chain of identical networks. A chain of three networks separated by dotted lines is shown.

and a prohibitively large number of accesses with vacuum feedthrough in the vacuum chamber. The problem is, therefore, how to realize the strap current distribution (i.e. constant $\left|I_{\mathrm{A}}\right|$ with the $\Delta \Phi$ inter-strap phasing with the minimum number of accesses to the machine. This is the subject of the next sections.

The study also indicates the large influence of the strap and antenna box geometry on the performances. This is mainly due to its influence on the mean strap inductance that determines the needed voltage to drive the strap current.

\section{Feeding solution (step 1): traveling wave along a resonant strap array}

Figure 13(a) shows an array of short-circuited straps of length $l_{\text {str }}<\lambda_{0} / 4$. This array can be considered as a system of coupled strip lines near their current antinode where the RF field excitation by the strap current is maximum. The mutual coupling is, therefore, mainly inductive and as first approximation, we only consider the mutual inductance $M$ between consecutive straps. Each strap is also tuned at $f=f_{0}$ by an external capacitor $C$ to the same frequency (i.e. we have $(L C)^{-1 / 2}=f_{0}$ ). The losses are here assumed to be due to radiation and are modeled by the series resistors $R$ shown in the figure. This chain of resonant straps can be considered as composed of identical two-port networks connected in cascade as shown in figure 13(b). It corresponds to a bandpass filter (see e.g. [13] sections 4.8 and 4.9) which is equivalent to a transmission

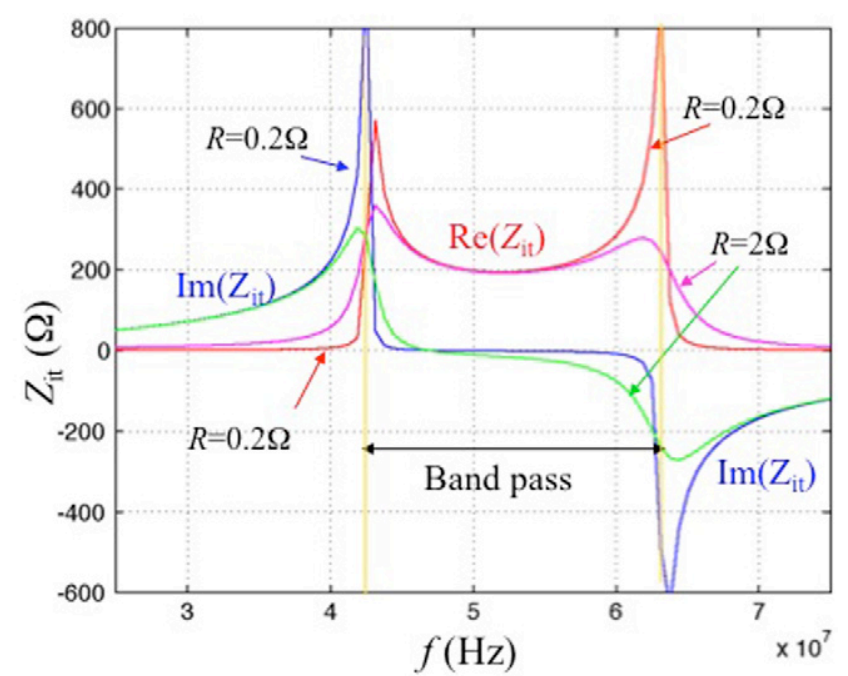

Figure 14. Iterative impedance $Z_{\mathrm{it}}$ as a function of frequency for two values of $R$ and for $L=127 \mathrm{nH}, M=48 \mathrm{nH}$ and $C=80 \mathrm{pF}$.

line section in its pass band characterized by its iterative impedance $Z_{\text {it }}$ (equivalent to the continuous line characteristic impedance) and the ratio of two consecutive strap currents $I_{n+1} / I_{n}=\exp (\mathrm{i} \Delta \Phi) . Z_{\text {it }}$ can be computed from the open circuit $Z_{\mathrm{oc}}$ or short-circuit $Z_{\mathrm{sc}}$ values of the two ports' elementary symmetrical network shown in figure $13(b)$ or by the terms of its $Z$ matrix by the relations $[13,14]$

$$
Z_{\mathrm{it}}=\left(Z_{\mathrm{oc}} Z_{\mathrm{sc}}\right)^{1 / 2}=\left(Z_{11}^{2}-Z_{12}^{2}\right)^{1 / 2} .
$$



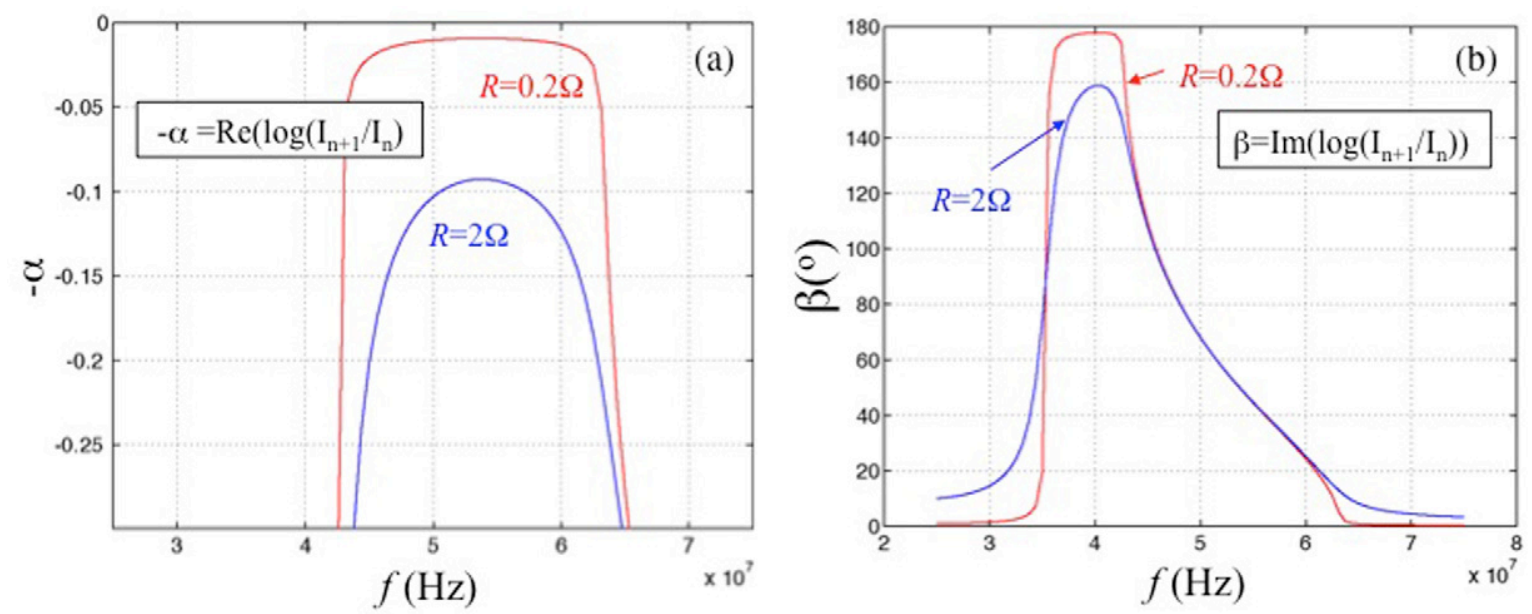

Figure 15. (a) and (b) Damping parameter $\alpha$ and phase angle $\beta$ between the current of two consecutive straps of the array.

The strap input current $I_{n}$ and voltage $V_{n}$ amplitudes are equal in the absence of losses $(R=0)$ and $\Delta \Phi$ as $Z_{\text {it }}$ are real. With losses $(R \neq 0), \Delta \Phi=\beta+\mathrm{i} \alpha$ is complex and, therefore, the strap current amplitudes are decaying as $\left|I_{n+1}\right| /\left|I_{n}\right|=\exp (-\alpha)$. Figure 14 gives an example of the evolution versus frequency of $Z_{\mathrm{it}}$ for two values of $R$ and figures $15(a)$ and $(b)$ show the corresponding curves of $\Delta \Phi=\beta+\mathrm{i} \alpha$. The main characteristics of this network are: (i) The damping parameter $\alpha$ increases with $R$ but the iterative impedance $Z_{\text {it }}$ and the phase $\Delta \Phi$ are weakly sensitive to the $R$ value if not too large. (ii) The phase $\Delta \Phi$ between two adjacent straps continuously varies in the pass band and can be adjusted by the choice of the frequency. (iii) If the array is terminated on its iterative impedance $\left(Z_{\text {out }}=Z_{\text {it }}\right)$ the input impedance $Z_{\text {in }}$ remains equal to $Z_{\text {it }}$ for any number of elementary networks in the chain. (iv) The traveling wave along the array may travel in both directions as for a continuous transmission line and will excite positive or negative $k_{z \text {,peak }}$ (v) This system is totally load resilient when terminated by a dummy load of impedance $Z_{\text {it }}$. The generator connected to input 1 then delivers a power $P_{\text {in }}=\left|V_{1}\right|^{2} /\left(2 Z_{\text {it }}\right)$ independent of the value of $R$. The power $P_{\text {in }}$ is shared between the power radiated by the straps and the power $P_{\text {out }}$ absorbed in the dummy load. (vi) The power radiated by the straps decays as a function of the strap number $n$ as $P_{n}=P_{1} \exp (-2 n \alpha)$ and the power delivered to the load $P_{\text {out }}=P_{\text {in }}-\Sigma_{n} P_{n}$. When the loading resistance $R_{\text {rad }}$ increases, the last straps of the array radiate less and less power. If $P_{\text {out }}$ is negligible, the last straps even constitute the TWA dummy load and an external load is no longer necessary. The expected radiated power gain due to the number of straps $n_{\text {str }}$ of the TWA section, therefore, drops by a factor $\left(1-\exp \left(2 n_{\text {str }} \alpha\right)\right) /\left\{n_{\text {str }}(1-\exp (2 \alpha))\right\}$ if the radiation $k_{z}$ spectrum is not deformed too much.

To radiate a large amount of power with good antenna efficiency a large number of straps are needed as explained in section 2.1, but they must be fed periodically to avoid too large a radiated power decay in the last straps of each TWA section. Another problem is the resonant tuning of each strap. We will address these problems in the next sections.

The system of a resonant strap array constitutes a combline. Comblines are commonly used in microwaves for bandpass filter application.
The use of a combline traveling-wave antenna was already considered for lower hybrid wave application [14] and has been proposed by the DIII-D team $[15,16]$ and successfully tested on JFT-2M [17] at $200 \mathrm{MHz}$ and $400 \mathrm{~kW}$ for fast wave current drive. The same system is presently considered [18] for very high harmonic fast wave current drive on DIII-D and FNSF-AT. The length of the straps is chosen to be at the $\lambda / 4$ or the $\lambda / 2$ resonance. In the JFT-2M experiment the 12-strap TWA was maintaining a good match to the impedance of the transmission line for all plasma modes including $\mathrm{H}$-mode, L-mode, Ohmic and vacuum. Measurements also indicated that unidirectional fast waves were successfully launched.

\section{Feeding solution (step 2): TWA sections mounted in a resonant ring}

In this section, we will consider an example of three sections of eight straps mounted in a resonant ring, but this case can be easily generalized to the case of $n_{\text {TWA }}$ sections of $n_{\text {str }}$ straps.

A similar concept is proposed in [19] where a 'ring antenna' all around the machine is operated as a fully internal resonant ring. A preliminary example of such a system, based on the ITER specifications, is presented in [20]. The system here discussed is quite different in the way it is assembled and operated, but it is based on the same principles.

\subsection{Computation of a TWA S matrix}

Figure 16 describes the antenna model of eight straps without loading solved numerically (MWS (CST MICROWAVE STUDIO $\left.{ }^{8}\right)$ modeling) to compute its $8 \times 8 S$ matrix. A similar simulation of a mock-up at $1 / 4$ scale was done to compare its computed $S$ matrix with the measured one. The comparison was made between an eight-strap mock-up and the equivalent 3D numerical model. Each strap is connected to a Type-N male connector, on both ends, that is clamped on a groove in the upper and lower side of the antenna box. This system allows the independent and arbitrary positioning of the strap with respect to the neighboring ones. Also, the lateral plates of the antenna 


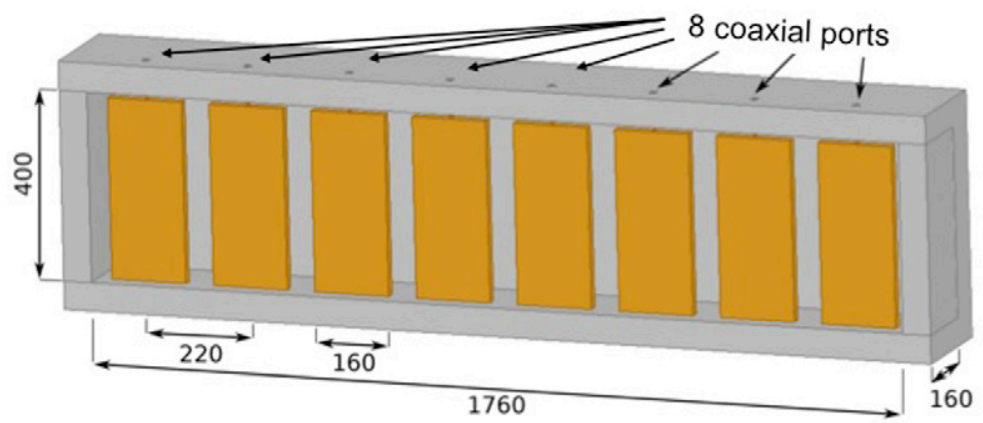

Figure 16. Model used for MWS simulation of an eight-strap TWA section in its antenna box. The strap tops are connected to the coaxial ports and their bottoms to the bottom of the box. The dimensions are expressed in $\mathrm{mm}$.

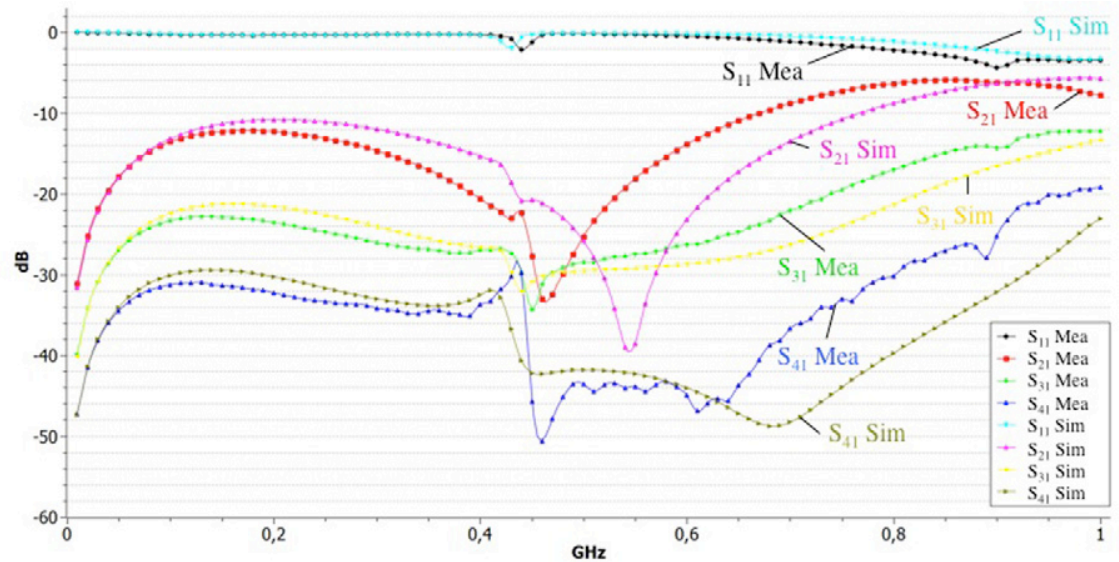

Figure 17. Comparison between mock-up simulation and measurement to validate the numerical modeling. $S$ matrix component with respect to port \#1 to evaluate the coupling with the first fourth neighboring elements and the self reactance.

box are movable in order to change the toroidal size of the box. To construct a combline structure, one of the two Type-N connectors is short circuited with an appropriate cap. The other end is then connected to the vector network analyzer by means of a cable. The $S$ matrix reference plane is set at the level of the connectors for both the measured and simulated devices.

The outcome of the comparison is presented in figure 17. There is good agreement between the simulated structure and the measured one in the low-frequency region (between 10 and $400 \mathrm{MHz}$ ). In the upper region, the response is a bit different, but a similar behavior of the structure is captured by the simulation. However, this part of the operating frequency region is not of interest for the system analyzed in this paper. The small difference in the response in the (low) frequency region of interest is due mainly to the fact that the $3 \mathrm{D}$ numerical model is more accurate in the geometrical details than the realized mock-up.

We then consider this a reliable modeling tool and we developed an ideal structure such that we can trust our simulation results from which the $8 \times 8 Z$ matrix giving the self $X(i, i)$ and all mutual $X(i, j)$ inductances of the straps can be deduced. We transform the $8 \times 8$ matrix into a $2 \times 2$ matrix between inputs $\# 1$ and $\# 2$ by terminating all straps by the same capacitive susceptance $B_{C}=\omega C$. The value of $C$ is chosen such that for the frequency $f=f_{0}=50 \mathrm{MHz}$ this susceptance cancels the mean strap self-inductance i.e. we take

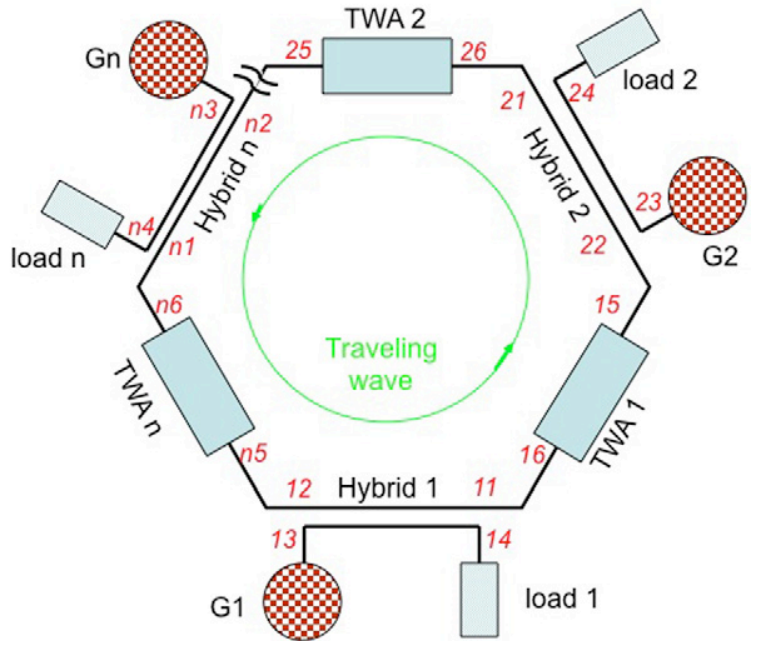

Figure 18. A number $n$ of TWA sections fed by hybrid junctions and mounted in a resonant ring circuit.

$$
C=\left\{\omega \sum_{i}[\operatorname{Im}(Z(i, i) / 8]\}^{-1} .\right.
$$

A resistance $R_{\text {rad }}$ in series with each strap circuit is introduced to simulate the radiation losses. From the obtained $2 \times 2$ matrix we can compute the iterative impedance by relation (6). In case of some asymmetry of the matrix, the iterative 

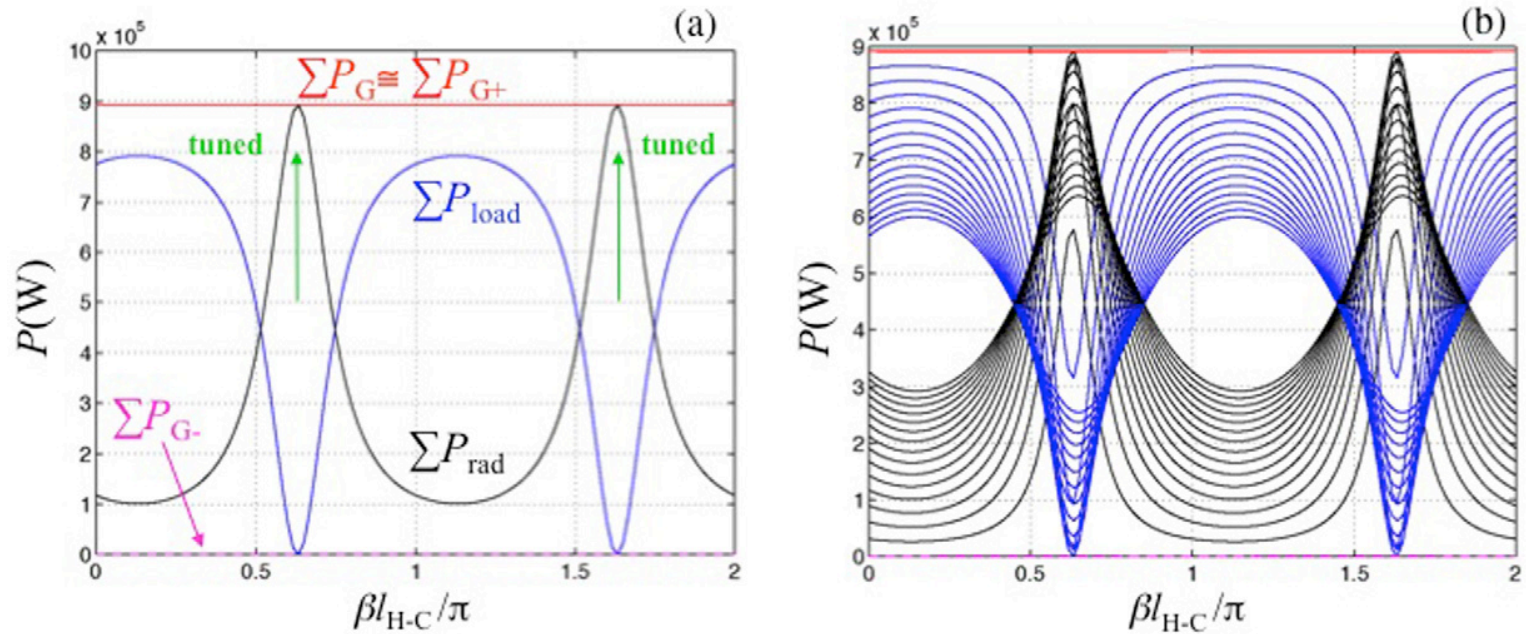

Figure 19. (a) Power balance versus normalized line length between hybrids and TWA section (at both sides) for $R_{\mathrm{rad}}=1.6 \Omega, f=50 \mathrm{MHz}$ and $\delta_{\mathrm{HYB}}=-3 \mathrm{~dB}$. (b) The same with different values of $R_{\mathrm{rad}}=(0.4,0.8,1.2, \ldots, 6) \Omega$.

impedance for both directions of propagation has to be computed [13].

\subsection{Feed of the TWA sections by hybrid junctions}

Figure 18 shows how the three considered TWA sections are mounted in a ring circuit fed by three quadrature hybrids. The $\mathrm{S}$ matrix of the hybrids $S_{\mathrm{HYB}}$ is given by the relation [14]

$$
S_{\mathrm{HYB}}=\left|\begin{array}{cccc}
0 & \mathrm{i} \gamma & \delta & 0 \\
\mathrm{i} \gamma & 0 & 0 & \delta \\
\delta & 0 & 0 & \mathrm{i} \gamma \\
0 & \delta & \mathrm{i} \gamma & 0
\end{array}\right|
$$

where the coupling of the hybrid is given by the parameter $\delta=\delta_{\mathrm{HYB}}$ and generally expressed in $\mathrm{dB}$. The symmetric matrix of the considered lossless hybrid is unitary and, therefore, the parameters $\gamma$ and $\delta_{\mathrm{HYB}}$ are linked by the relation $\gamma=\left(1-\delta_{\mathrm{HYB}}^{2}\right)^{1 / 2}$. The port $\# 3$ of each hybrid is connected to a generator $G_{n}(n=1,2,3)$ and its port \#4 to a dummy load. The TWA sections are connected to ports \#1 and \#2. The ring circuit is fed by the forward voltages of the three generators $V_{\mathrm{G} i+}$, which are taken equal in amplitude and by symmetry with a phase difference of $\pm 120^{\circ}$ between them. We also choose hybrids having output lines with characteristic impedance equal to the iterative one of the TWA sections. Then, all voltages and current along the ring (i.e. in figure 18 at positions $l, m$ with $l=1$ to 3 and $m=1$ to 4 ) and inside the TWA sections are computed by connecting the hybrid matrices to the $2 \times 2$ TWA ones and going back from the $2 \times 2$ to the $8 \times 8$ TWA matrices. The length of the lines connecting the hybrids to the TWA sections is assumed equal and can be adjusted.

\subsection{Resonant ring condition and properties}

The resonant ring condition corresponds to the maximum power transfer from the generators to the TWA sections. It is adjusted by the electrical length $l_{\mathrm{H}-\mathrm{C}}$ between the hybrids and

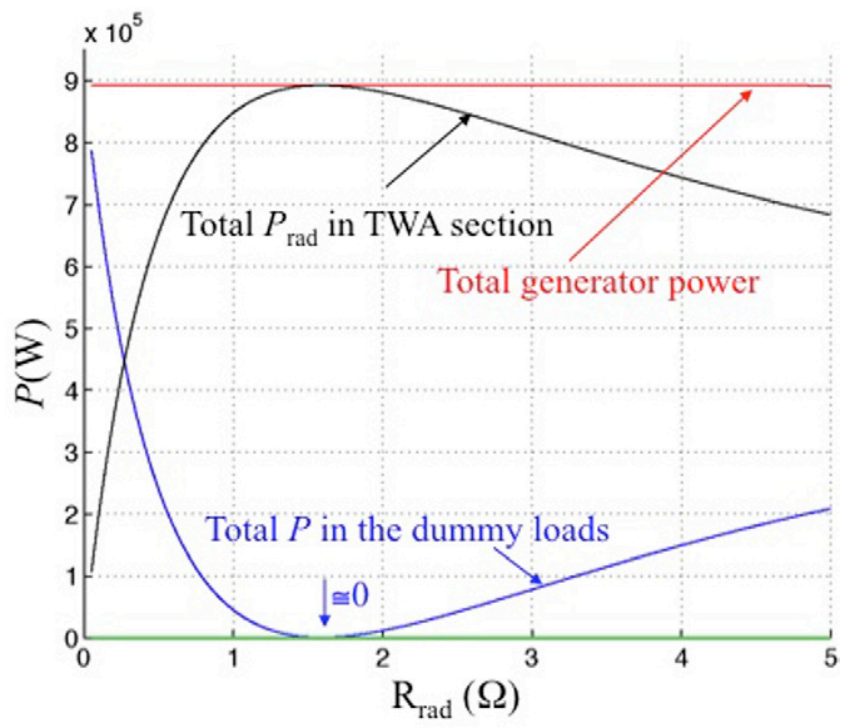

Figure 20. Power balance versus radiation resistance with $\delta_{\mathrm{HYB}}=-3 \mathrm{~dB}$ and $f=50 \mathrm{MHz}$. The reflected power at generators and TWA sections is almost zero.

the TWA sections. The power balance is shown in figure 19(a) for $f=50 \mathrm{Mhz},\left|V_{\mathrm{G} i+}\right|=10 \mathrm{kV}, R=1.6 \Omega$ and $\delta_{\mathrm{HYB}}=-3$ $\mathrm{dB}$ as a function of $l_{\mathrm{H}-\mathrm{C}}$. For these parameters the total power dumped in the dummy loads vanishes and the one delivered to the TWA sections corresponds to the total forward power $\Sigma_{i}$ $P_{\mathrm{G} i+}$ of the three generators at ring resonance. This resonance occurs for discrete values of $l_{\mathrm{H}-\mathrm{C}}$ such that the phase angle difference for the traveling wave circulating in one direction along the ring is equal to $p(2 \pi)$ with $p=0,1,2,3 \ldots$ We have the following properties: (i) at ring resonance all TWA sections are terminated on their iterative impedance, (ii) the generators (assumed matched on the characteristic impedance of the hybrids) receive no significant reflected power $P_{\mathrm{Gi} i}$, (iii) the voltage standing wave ratio (VSWR) in the ring equals 1 due to the absence of a significant traveling-wave component in the opposite direction, (iv) the $l_{\mathrm{H}-\mathrm{C}}$ adjustment for the resonant ring condition remains valid for a large range of 

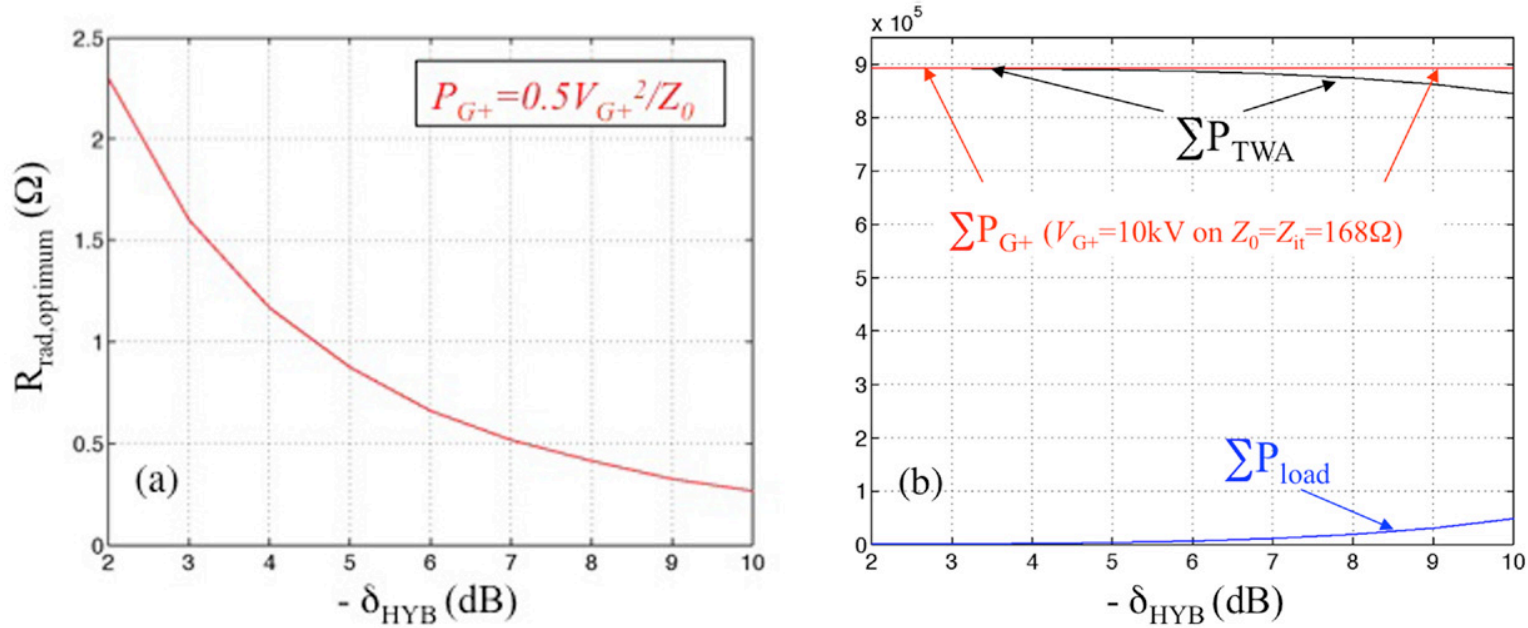

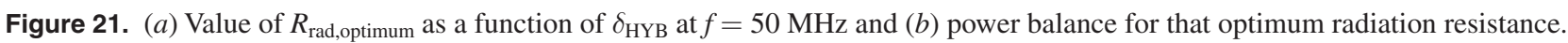
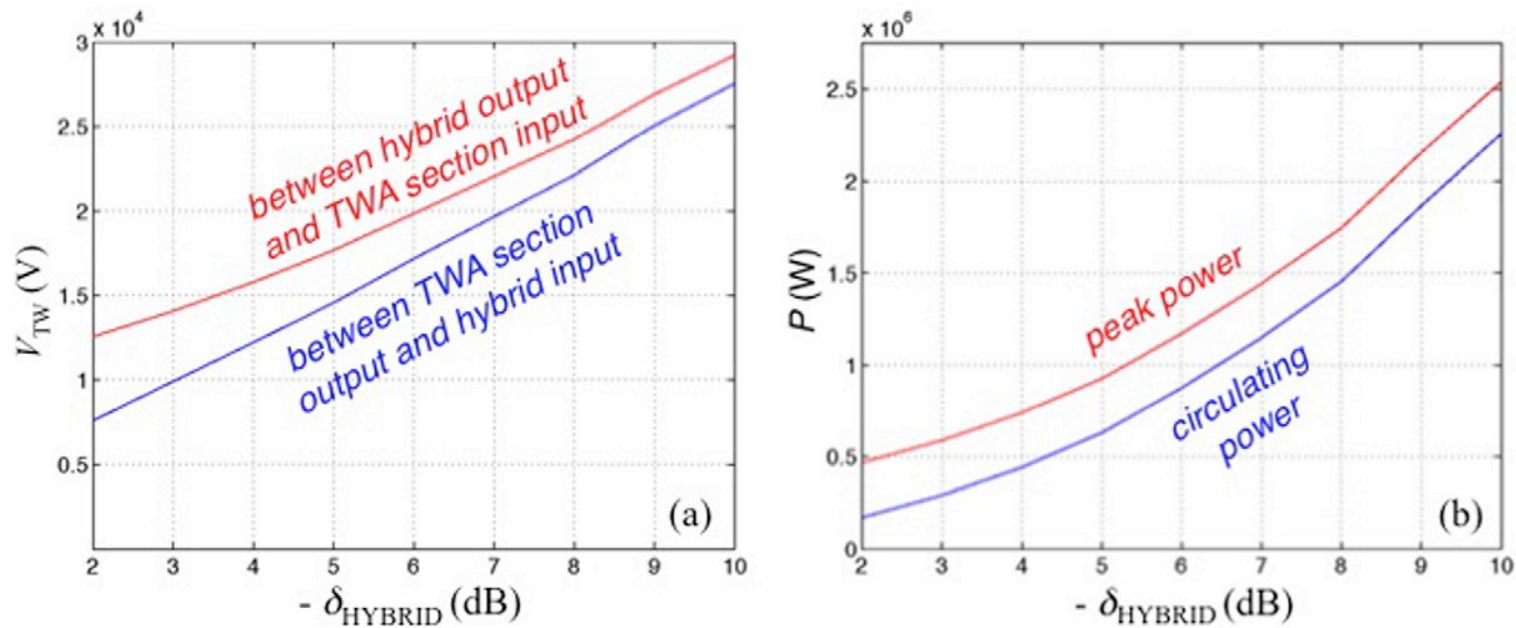

Figure 22. (a) Voltage of traveling wave all along the resonant ring of figure 18 for $R_{\text {rad }}=R_{\text {rad,optimum, }} f=50 \mathrm{MHz}$ and $\left|V_{\mathrm{G}+}\right|=10 \mathrm{kV}$. (b) Corresponding peak and circulating power in the ring.
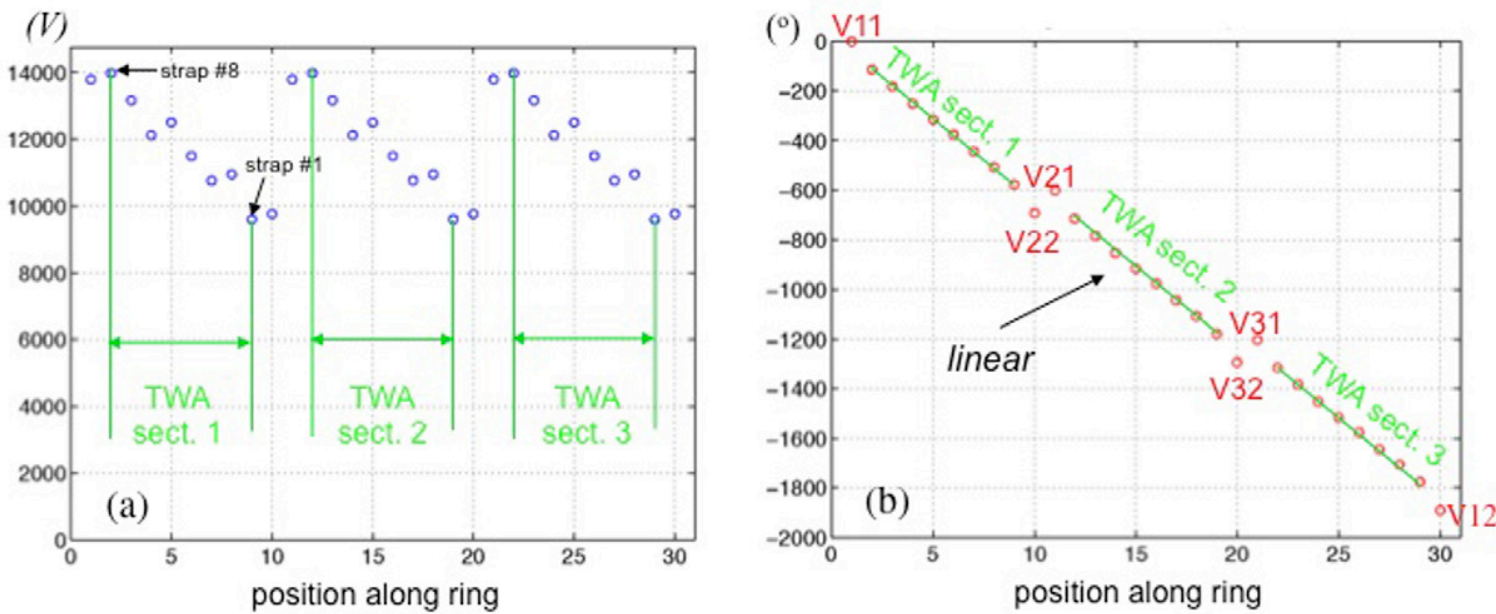

Figure 23. (a) Voltage amplitude at input and output of hybrids and at the eight straps of the TWA sections of figure 18 for $R_{\text {rad }}=1.65 \Omega$, $f=50 \mathrm{MHz},\left|V_{\mathrm{G}+}\right|=10 \mathrm{kV}, \psi_{\mathrm{G}}=120^{\circ}$ and the hybrid coupling of $-3 \mathrm{~dB}$. (b) Corresponding phase angle of the voltage where the total angle around the ring is $10 \pi$. The numbering of $V$ gives the locations indicated in figure 18 . 

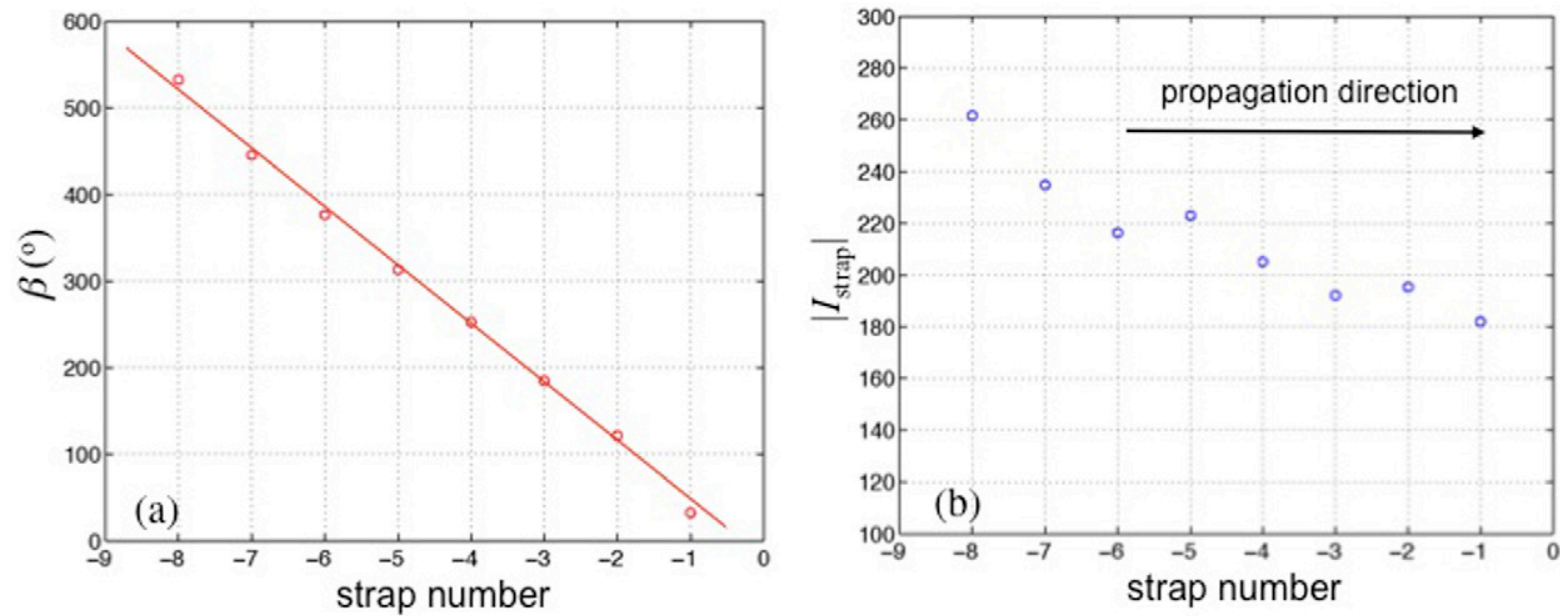

Figure 24. (a) Phase of strap current in one TWA section for the conditions of figure 23 and $(b)$ corresponding strap current amplitude. The mean phase difference: $\Delta \beta=66.15^{\circ}$ and there is almost linear phase variation of the current along the combline.

radiating resistances as shown in figure 19(b), (iv) the system is load resilient, because the delivered power per generator $P_{\mathrm{G} i}$ $\cong P_{\mathrm{G} i+}=0.5\left|V_{\mathrm{G} i+}\right|^{2} / Z_{0}$ does not depend on $R_{\mathrm{rad}}$ but from the line characteristic impedance $Z_{0}=Z_{\mathrm{it}}$. This power is shared between the TWA and the load.

\subsection{Maximum performances of the resonant ring}

In the curves of the $R_{\text {rad }}$ scan of figure $19(b)$ we see that the generator power is only partly transferred to the TWA at resonance when $R_{\mathrm{rad}} \neq 1.6 \Omega$, the remaining part of the generator power being dumped in the dummy load. In fact, this is due to the choice of the hybrid coupling value $\delta_{\mathrm{HYB}}=-3 \mathrm{~dB}$ for which $R_{\text {rad }}=R_{\text {rad,optimum }}=1.6 \Omega$. This appears more clearly in the $R_{\text {rad }}$ scan of figure 20 where the total power in the loads and the total power in the TWAs are displayed as a function of $R_{\mathrm{rad}}$ for $\delta_{\mathrm{HYB}}=-3 \mathrm{~dB}$. Figure 21(a) shows the value of $R_{\text {rad,optimum }}$ as a function of the hybrid coupling $\delta_{\text {HYB }}$. Figure 20 shows that a large fraction of the generator power is delivered to the TWA for a broad range of $R_{\mathrm{rad}}$ around $R_{\text {rad,optimum }}$ (more than $93 \%$ from 0.9 to $2.8 \Omega$ in our example) but it is best to use hybrid junctions with adjustable coupling. Figure 21(b) shows the best performances achievable for power transfer to the TWA sections when $\delta_{\text {HYB }}$ is adjusted to have the radiation resistance equal to its optimal value as given in figure 21(a). With this adjustment almost all generator power remains coupled to the TWA sections in a large domain of $R_{\text {rad. }}$. Only for small $R_{\text {rad }}(<0.5 \Omega)$ requesting large values of $-\delta_{\text {HYB }}$ ( eight in our example) a small part of the power is absorbed in the dummy loads.

The voltage amplitude along the traveling wave as a function of $\delta_{\mathrm{HYB}}$ is shown in figure 22(a) for the optimal condition $R_{\text {rad }}=R_{\text {rad,optimum. As the voltage amplitude decreases }}$ along the TWA sections due to the radiative losses, its value is lower between the TWA section output and the hybrid. The corresponding active power $\left(=|V|^{2} /\left(2 Z_{\mathrm{it}}\right)\right)$ is displayed in figure 22(b). The difference between the two curves is the power that is injected in the ring by each hybrid and radiated afterwards by the next TWA section. The lower curve

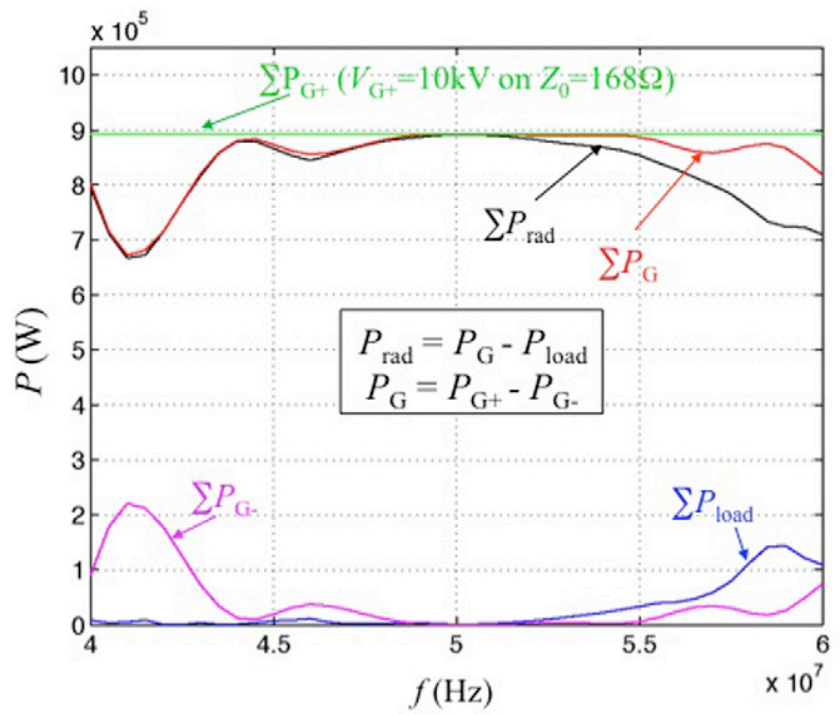

Figure 25. Power balance of the resonant ring of figure 18 as a function of the frequency. The ring is put at resonance at each frequency by the adjustment of $l_{\mathrm{H}-\mathrm{C}}$ (see figure $26(a)$ ).

corresponds to the circulating power in the resonant ring. The voltage amplitudes and, consequently, the corresponding amount of power increases when $R_{\text {rad }}=R_{\text {rad,optimum to main- }}$ tain the radiated power of each TWA section equal to the delivered power per generator, which only depends on $\left|V_{\mathrm{G} i+}\right|$ as stated in section 4.3.

\section{5. $V$ and I amplitude and phase along the resonant ring}

Figure 23(a) gives an example of the voltage amplitude on the inputs and outputs of the hybrids and the eight straps of the TWA sections (the waves travel from strap \#8 to strap \#1 in each section) at ring resonance (case $R_{\mathrm{rad}}=R_{\mathrm{rad}, \mathrm{optimum}}=1.6 \Omega$ with $\delta_{\mathrm{HYB}}=-3 \mathrm{~dB}$ as indicated for $\beta l_{\mathrm{H}-\mathrm{C}} / \pi=0.6$ in figure 19(a)). The voltage decays in each section of the TWA due to radiative loss in $R_{\text {rad }}$. The corresponding behavior of the voltage phase angle is displayed in figure 23(b). The phase angle decreases linearly with the position in the TWA 

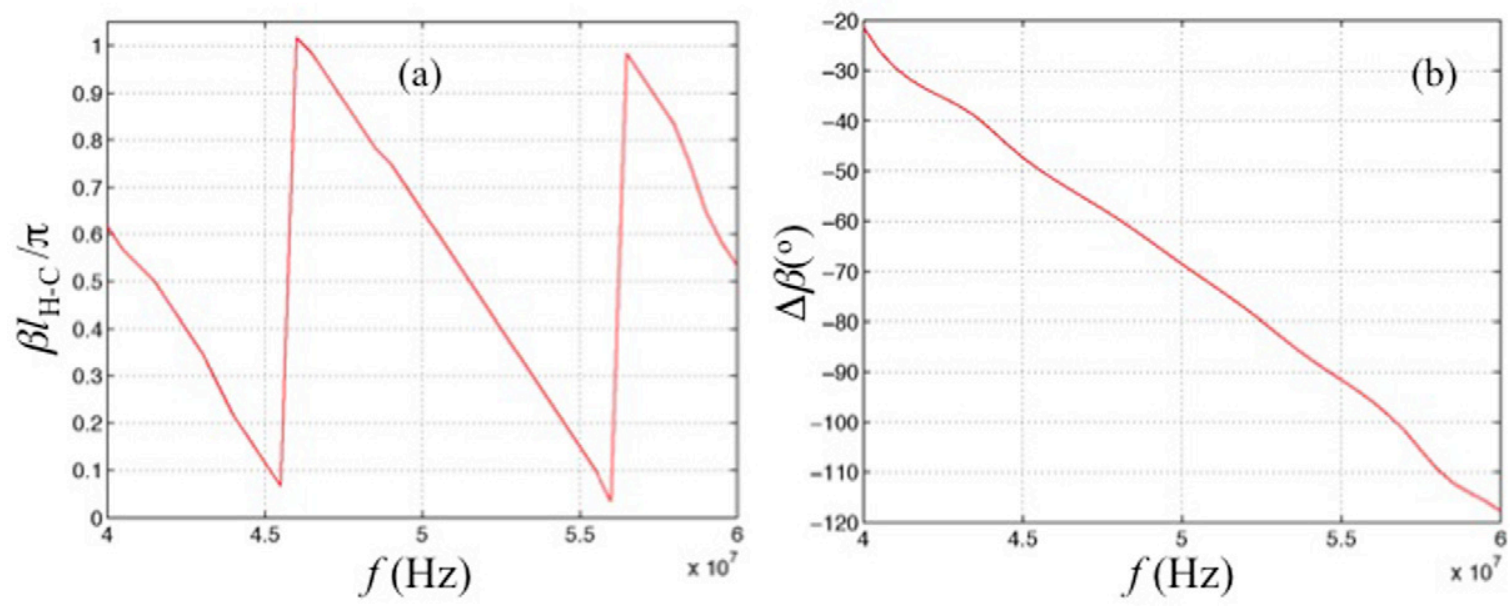

Figure 26. (a) Normalized distance TWA section-hybrid versus $f$ to bring the ring at resonance. $(b)$ Mean angle difference between two consecutive straps of the TWA sections versus $f$.

sections, because the angle $\Delta \Phi$ between two consecutive straps remains constant as in the simplified model of section 3. The corresponding similar behavior of the strap current phase and amplitude is show in figures 24(a) and $(b)$.

The total angle variation along the resonant ring is $p(2 \pi)$ with $p=0,1,2,3 \ldots$ as already stated in section 4.3. This restricts the possible phase differences between the generators. For our case, with all distances $l_{\mathrm{H}-\mathrm{C}}$ being taken the same, the phase angle difference between the generators must be $m(2 \pi) / n_{\text {TWA }}$ where $n_{\text {TWA }}$ is the number of TWA sections and $m=0,1,2,3 \ldots$

\subsection{Frequency dependence}

The resonant ring remains operational in a frequency domain around $f=f_{0}=50 \mathrm{MHz}$. The power balance as a function of the frequency $f$ shows this in figure 25. Except for the frequency and the distance $l_{\mathrm{H}-\mathrm{C}}$ the parameters are the same as for figure 19(a). The performances remain very good in the range $45 \mathrm{MHz}<f<55 \mathrm{MHz}$ characterized by very low reflected power at generators $P_{\mathrm{G} i-}$ and dumped power in the dummy loads $P_{\text {load }}$. Outside this band, $P_{\mathrm{Gi} i-}$ and/or $P_{\text {load }}$ are becoming non-negligible as can also be seen in figure 25 . An adjustment of the distance $l_{\mathrm{H}-\mathrm{C}}$ is needed for each frequency to remain in the resonant ring conditions (see figure 26(a)). As expected from section 3 the phase difference $\beta$ between two consecutive straps varies with the frequency as illustrated in figure $26(b)$ and can, therefore, be adjusted by it.

\section{Consistent plasma-coupling computation with resonant-ring TWA excitation}

\subsection{Case of one TWA section}

The model of the resonant ring is linked to the coupling code ANTITER II in order to determine the $R_{\text {rad }}$ value, the best corresponding hybrid coupling parameter $\delta_{\mathrm{HYB}}$ and the resulting radiated power for a given value of the applied forward voltage $\left|V_{\mathrm{G} i+}\right|$ at port $\# 3$ of the hybrids. The result is obtained by iterations: a guess value is first chosen for $R_{\mathrm{rad}}$ that determines the best $\delta_{\mathrm{HYB}}$ choice. The resonant ring model then computes the strap current distribution in the TWA section that is introduced in the ANTITER code. A new value of $R_{\text {rad }}$ is then obtained and the procedure is iterated until the new values of $R_{\text {rad }}$ and radiated power given by ANTITER are converging to the ones used or computed in the resonant ring model.

The convergence with the '2010-low' plasma profile is obtained for $R_{\text {rad }}=0.6 \Omega$ and $\delta_{\mathrm{HYB}}=-7 \mathrm{~dB}$ (value in accordance with figure $21(a)$ ). For $\left|V_{\mathrm{G} i+}\right|=10 \mathrm{kV}$ and in the absence of reflected power the radiated power by the three considered TWA sections depends only on $\operatorname{Re}\left(Z_{\mathrm{it}}\right)$ and amounts $0.9 \mathrm{MW}$ as seen in figure 25 . The resulting phase and amplitude of the current $I_{\mathrm{A} i}$ of the eight straps of one TWA section are shown in figure $27(a)$. The strap phasing leads to $k_{z 0} \cong 5 \mathrm{~m}^{-1}$ as shown by the $k_{z}$ power spectrum given in figure $27(b)$. It corresponds to a radiated power of $0.3 \mathrm{MW}$ for one TWA section. The current decay along the TWA section is small due to the low value of $R_{\text {rad }}$.

The excitation and penetration of the wave into the plasma bulk is obtained in the real space by Fourier inversion of its Fourier $k_{z}, k_{y}$ spectrum and is illustrated in figures 28(a) and (b). These figures respectively give a contour plot of $\operatorname{Re}\left(E_{y}\right)$ and $\operatorname{Re}\left(\omega B_{z}\right)$ in the horizontal mid-plane of one TWA section. The $z$-axis is along the toroidal direction, the $x$-axis is the radial one with its origin located at the antenna front and the plasma profile of figure 3 is located in the region $x<0$. Near $x=0$ one observes the wave propagating along the TWA towards the $z$-axis. This wave characterized by $\left|k_{z}\right| \cong\left|k_{z 0}\right|>k_{0}$ is evanescent in the radial direction until the $x$ position $(x=-0.156$ $\mathrm{m})$ where the electron plasma density reaches the cutoff value $N_{\mathrm{CO}}\left(k_{z 0}\right)\left(1.54 \times 10^{18} \mathrm{~m}^{-3}\right)$. There, the propagation of the magneto-sonic wave starts and appears on the figure with its constant phase surface at a given time $t$ and for $y=0$ determined by $k_{z 0} z+k_{y 0} y+k_{x} x=\omega t$ with $k_{x}^{2}=k_{\perp}^{2}-k_{y 0}^{2}$ and $k_{\perp}^{2}=$ $k_{0}^{2} \varepsilon_{1}-k_{z 0}^{2}-k_{0}^{4} \varepsilon_{2}^{2} /\left(k_{0}^{2} \varepsilon_{1}-k_{z 0}^{2}\right) . \varepsilon_{1}$ and $\varepsilon_{2}$ are, respectively, the $(1,1)$ and $(1,2)$ terms of the cold dielectric plasma tensor and $k_{y 0} \cong 0$ as can be seen in figure $27(b)$. Starting from the antenna 

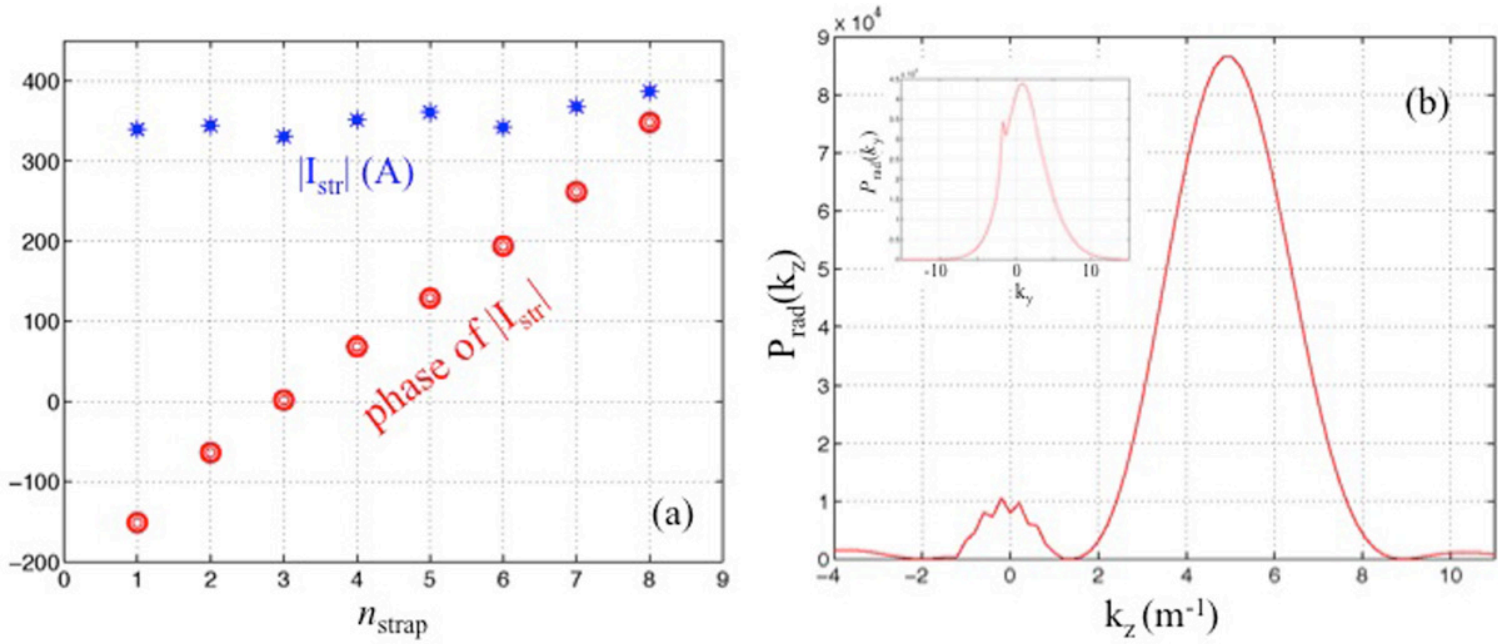

Figure 27. (a) Radiating strap current distribution of the TWA section of figure 16 at $50 \mathrm{MHz}$ for $R_{\mathrm{rad}}=0.6 \Omega \mathrm{when} V_{\mathrm{G}+}=10 \mathrm{kV}$. (b) Corresponding radiated $k_{z}$ and $k_{y}$ power spectra.

(a)

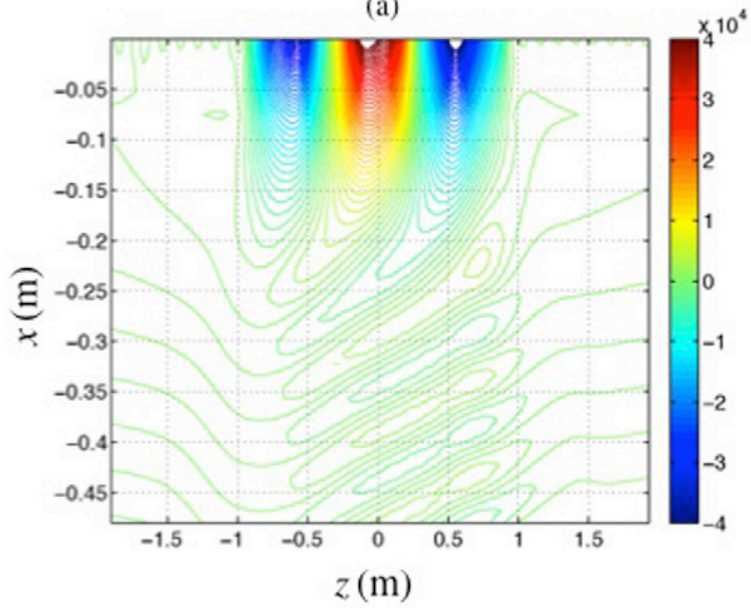

(b)

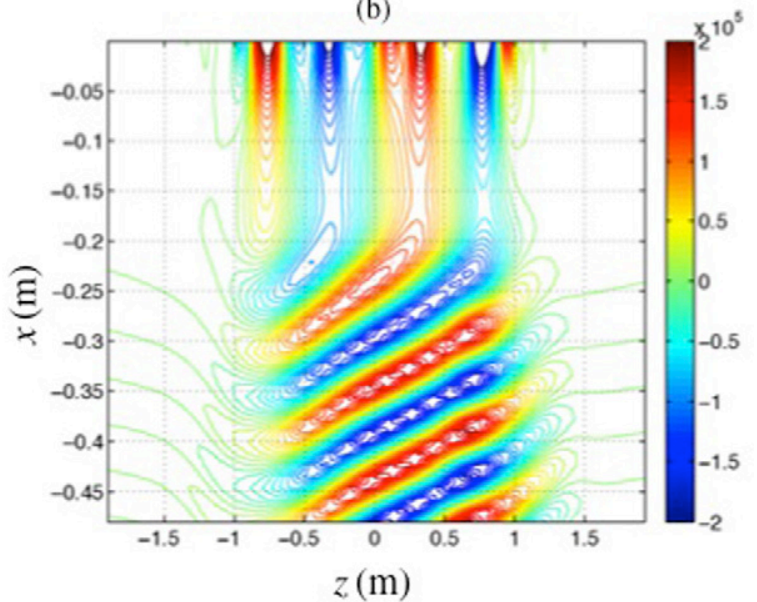

Figure 28. Contour plot of $(a) \operatorname{Re}\left(E_{y}\right)$ and $(b) \operatorname{Re}\left(\omega B_{z}\right)$ at $t=0$ in the $(x, z)$ plane at $y=0$ starting from the front of the TWA section of figure 16 in $x=0$ to deep in the plasma profile of figure 3 (D-T plasma, $50 \mathrm{MHz}$, separatrix at $x=-23 \mathrm{~cm}$ where $B_{\text {sep }}=2.9 \mathrm{~T}$ ). The radiating strap current distribution is shown in figure 27(a).

front, both the amplitudes of $E_{y}$ and $H_{z}$ decrease first in the evanescence region. For $N>N_{\mathrm{CO}} H_{z}$ starts to increase even to a value exceeding the one at the antenna front, whereas $E_{y}$ continues to decrease as shown when comparing figure 28(a) with 28(b). This is due to variation of wave impedance $E_{y} / H_{z}$ $\cong \omega \mu_{0} \xi\left(k_{z 0}, k_{y 0}\right)$ with the plasma density (at large $N$ we have $|\xi| \cong 1 /|k \perp| \cong V_{\mathrm{A}} / \omega, V_{\mathrm{A}}$ being the Alfven velocity).

\subsection{Case of several uniformly spaced TWA sections}

What is striking is the fact that the region of the magneto-sonic wave propagation inside the plasma in front of each TWA section remains roughly limited to the width of the antenna with some slight bending in the direction of wave propagation and that the region outside it is only slightly perturbed. This is well illustrated in figure 6. Then, if the distance between two consecutive TWA sections is not very short, each section seems to act independently. Figure 29(a) shows contour plot of $\operatorname{Re}\left(\omega B_{z}\right)$ for three TWA sections with the same parameters and plasma profile as figure $28(b)$. The phasing of the three sections is identical and the distance $T$ along the $z$-axis between the centers of two adjacent TWA sections is their spatial period and is equal to $3.73 \mathrm{~m}$ (i.e. twice the length of a TWA section box). The total radiated power of the three sections is, as expected, equal to three times the radiated power of the single TWA section of figure 28 with less than $0.5 \%$ error. The $k_{z}$ power spectrum is, on the other hand, modified by the periodicity of the TWA sections. Figure 29(b) shows the resulting spectrum $P_{n \text { BОх }}\left(k_{z}\right)$ compared to the one of the single box $P_{\text {single }}\left(k_{z}\right)$ multiplied by $n_{\mathrm{BOX}}^{2}$ i.e. by $9 . P_{n \mathrm{BOX}}\left(k_{z}\right)$ has three main peaks enclosed and their amplitude is limited by $n_{\mathrm{BOX}}^{2} P_{\text {single }}\left(k_{z}\right)$. These peaks occur for $k_{z}=p 2 \pi / T$ for the integer values of $p$ lying inside the domain limited by $n_{\mathrm{BOX}}^{2} P_{\text {single }}\left(k_{z}\right)$ : here for $p=2,3$ and 4. This behavior follows from the properties of the Fourier transforms. We show now a case of interacting TWA sections with their relative phasing adjusted to produce a continuous wave front in the plasma. The distance $T=1.91 \mathrm{~m}$ exceeds by $5 \%$ the TWA section box length. Figure 30(a) 

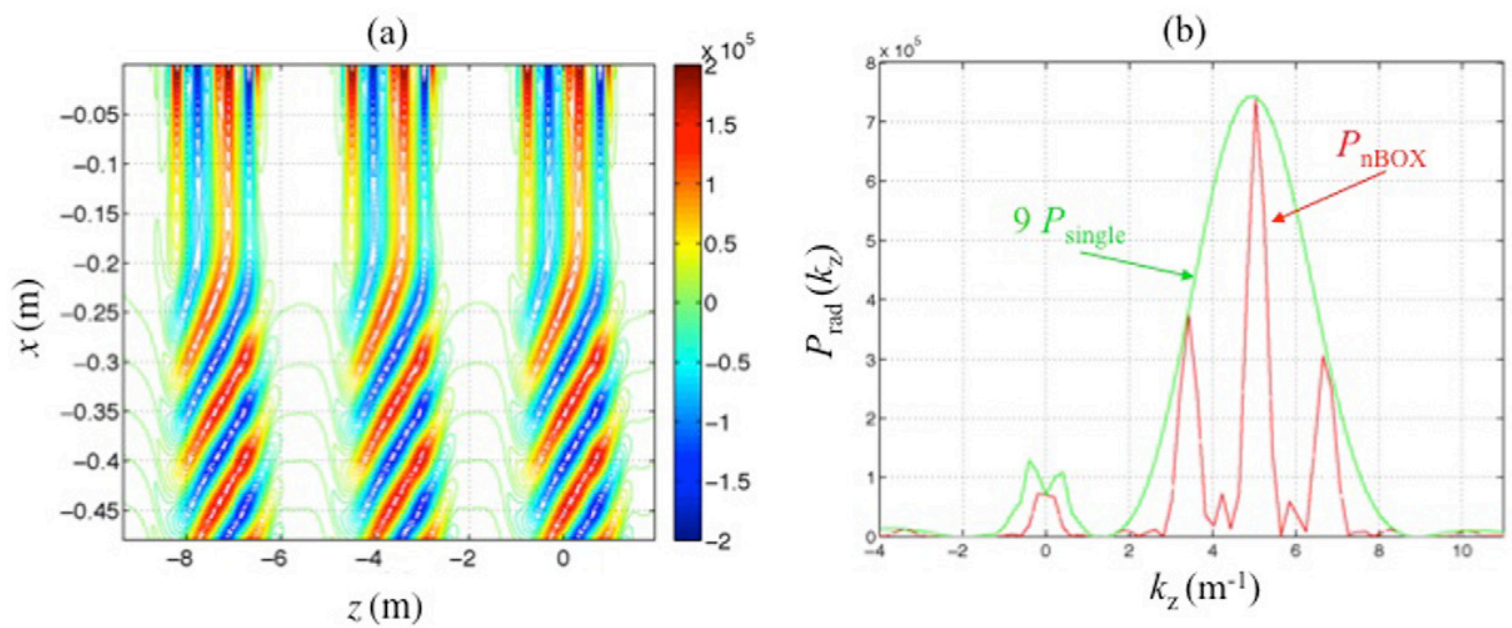

Figure 29. (a) Contour plot of $\operatorname{Re}\left(\omega B_{z}\right)$ at $t=0$ in the $(x, z)$ plane at $y=0$ starting from the antenna fronts in $x=0$ up to deep in the plasma profile of figure 3. Three TWA sections (each as shown in figure 16) are exciting the plasma with the strap current distribution shown in figure 27(a). There is no phasing difference between the three sections and the conditions are the same as for figure 28.

(b) Radiated power $k_{z}$ spectrum of a single TWA box $P_{\text {single }}$ multiplied by $n_{\mathrm{BOX}}^{2}$ compared to the one of three TWA boxes $P_{n \mathrm{BOX}}$.

(a)

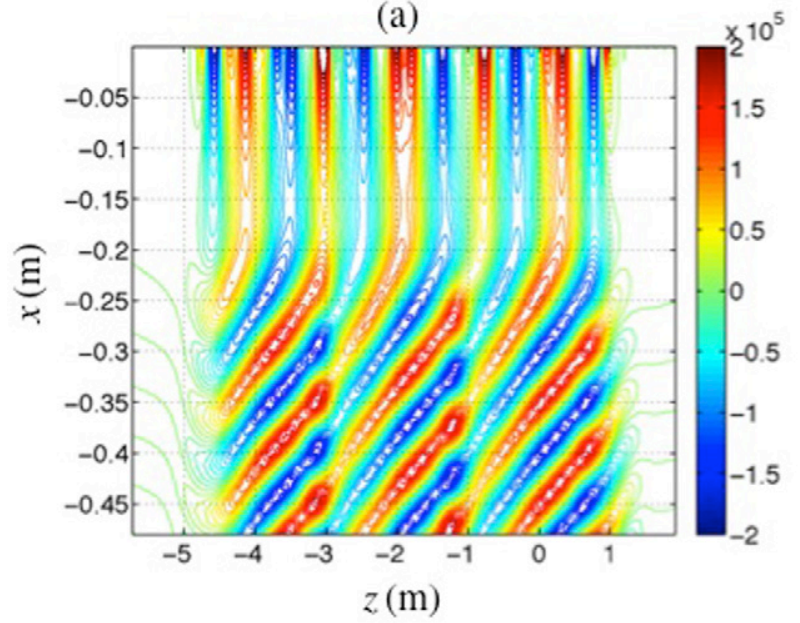

(b)

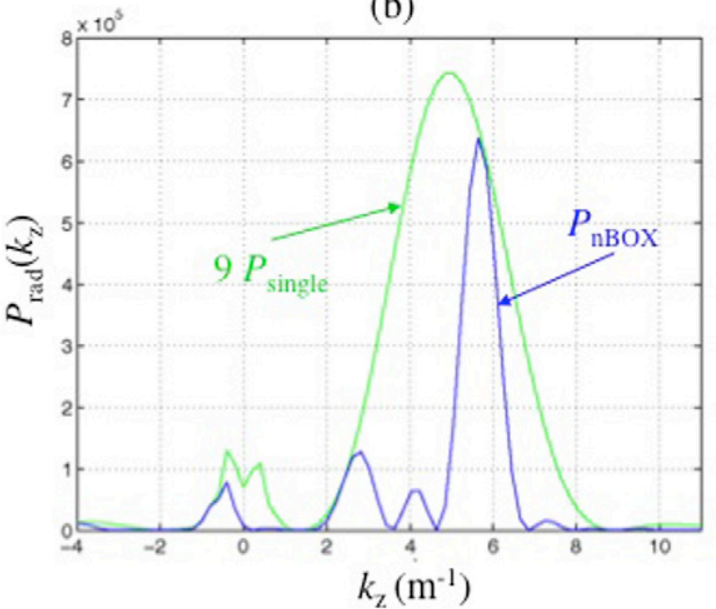

Figure 30. (a) Contour plot of $\operatorname{Re}\left(\omega B_{z}\right)$ at $t=0$ in the $(x, z)$ plane at $y=0$ starting from the antenna fronts in $x=0$ up to deep in the plasma profile of figure 3 . Three contiguous TWA sections (each as shown in figure 16) are exciting the plasma with the strap current distribution shown in figure 27(a). There is a phasing difference between the power sources of the three sections in order to have phase increment continuity in the three sections. The conditions are the same as for figure 28. (b) Radiated power $k_{z}$ spectrum of a single TWA box $P_{\text {single }}$ multiplied by $n_{\mathrm{BOX}}^{2}$ compared to the one of three TWA boxes $P_{n \text { BOX }}$.

displays the resulting contour plot of $\operatorname{Re}\left(\omega B_{z}\right)$. The power refueling of each TWA box appears clearly on the wave front amplitude. The total radiated power is still equal to three times the radiated power of the single TWA section of figure 28 with less than $1 \%$ error. The system is no longer periodic due to the different phasing of each TWA section, but the spectrum is still made of peaks limited by the function $n_{\mathrm{BOX}}^{2} P_{\text {single }}\left(k_{z}\right)$ as shown in figure $30(b)$. Their $k_{z}$ occurrence is still to be investigated. Note also that possible effects due to mutual coupling between the different TWA sections, particularly for this last case, are neglected.

As already pointed out in section 1 the use of ANTITER II iterated with the TWA ring model allows a fast computation on a laptop of all wave and circuit characteristics of the complete
TWA system. Only the numerical modeling of the $S$ matrix of the TWA section without simplified geometry requires more computing time and hardware. The modeling by TOPICA [9] of the TWA section in front of the plasma profile would avoid the iteration because the $S$ matrix of the TWA would include the losses due to the radiation in the plasma. This tool requires many more computing capabilities, but must certainly be used to check the results of our fast procedure in the last steps of the design.

\section{Important points for practical application}

In this section, we will present three main important points to be considered for a practical application of the TWA concept for the design of an antenna system. This design has to be 


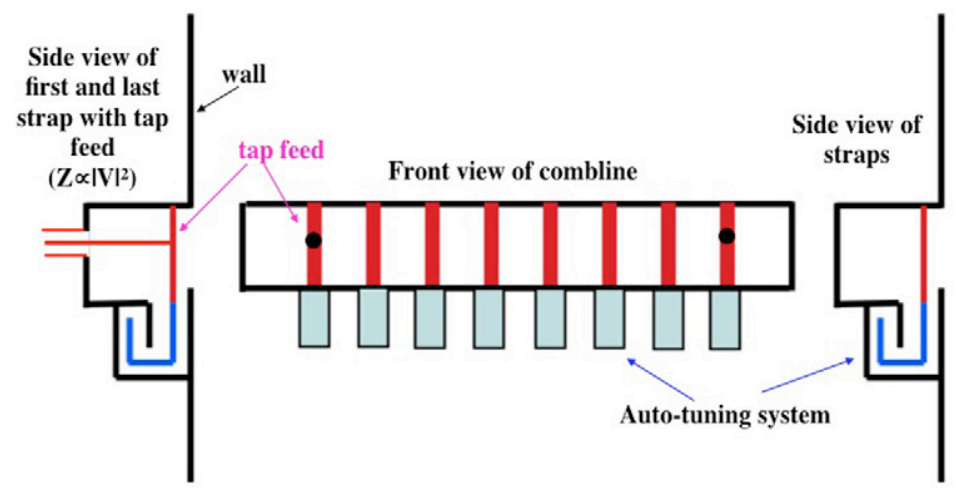

Figure 31. Section of TWA with tap feed on the first and last strap and with tuning capacitance at each strap.

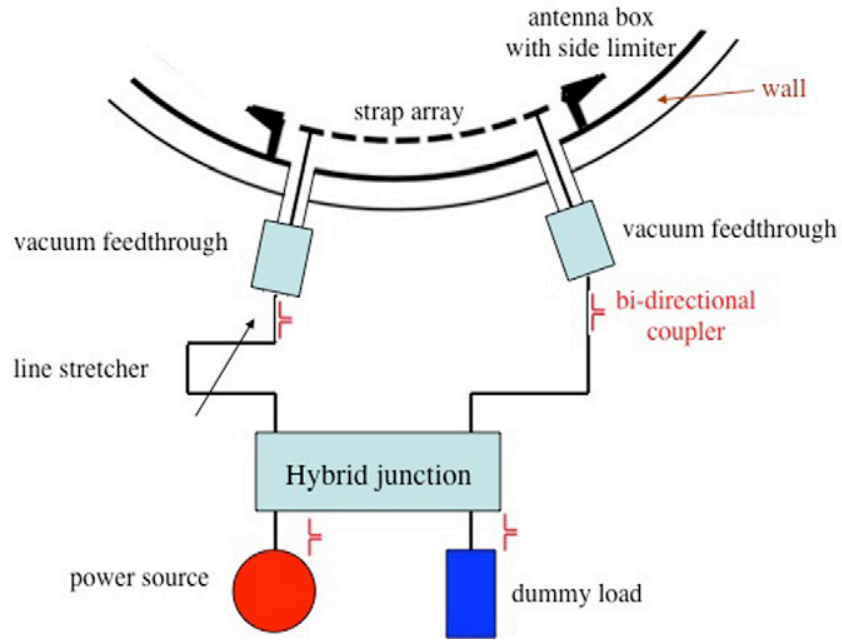

Figure 32. Resonant ring with single TWA section adjusted by the line stretcher. In case of adjacent line sections, the side limiters are replaced by the first or last strap of the neighboring section.

integrated in the wall of the tokamak and requires from the RF engineers a close collaboration with the machine design team.

\subsection{Value of iterative impedance}

For the TWA section of figure 16 the iterative impedance $Z_{\text {it }} \cong$ $168 \Omega$ and this value is used for the characteristic impedance of all the ring components. This value is not at all standard for the RF components and does not correspond to the best choice for power dissipation and voltage standoff. It is possible to bring down $Z_{\text {it }}$ to a standard value like $50 \Omega$ by tap connection at the first and last strap of each TWA section, as shown in figure 31. Indeed the tap acts as an impedance transformer with $Z_{\text {TAP }}$ proportional to $\left|V_{\text {TAP }}\right|^{2}, V_{\text {TAP }}$ being the strap voltage at the tap position.

\subsection{Strap-tuning capacitance}

The number of vacuum feedthroughs must be restricted to the connections with the hybrids. Therefore, the tuning capacitance of each strap must be integrated in the TWA line

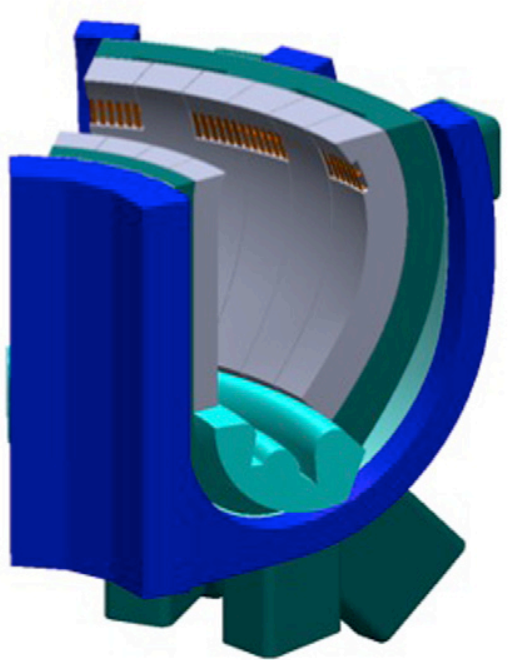

Figure 33. Artist's view of DEMO showing one complete TWA section and two halves of a system of 18 TWA sections installed all around the torus.

sections, i.e. the straps must be auto-tuned by design. A conceptual proposal is shown in figure 31 where each strap is connected to a folded open line section providing the needed capacitance. The design must take care that sufficient voltage standoff is maintained in this folded line. This $V$ standoff is the main limiting factor of the power capability.

\subsection{Choice of single or multiple resonant ring(s)}

Another possible multi-TWA section configuration is depicted schematically in figure 32 . Each TWA section is independently fed by its own power source in its own resonant ring tuned by a line-stretcher. The phasing between the power sources adjusts the one between the TWA sections. The two configurations (figures 18 and 32) are functionally equivalent, but the second is more effective in terms of reliability. Indeed, in case of a failure of one TWA section, only the corresponding resonant ring is affected, while with the first configuration the whole system will be affected. 


\section{Conclusions}

From our study we can draw the following conclusions:

- ICRF heating and/or current drive of DEMO can be excited by sections of TWA mounted in one or several resonant ring circuit(s) fed by hybrid junctions.

- At ring resonance, conditions with almost total power radiation to the plasma and negligible absorption in the loads are found for a broad domain of loading resistance and frequency.

- The generators remain almost matched for all loading conditions and, therefore, the system is totally load resilient. The TWA voltage adjusts itself to deliver the total power provided by the generators to the plasma.

- The radiation power is very selective in its toroidal propagation coeffient $k_{z}$ with the maximum radiation taking place for or near $k_{z 0}=\operatorname{Re}(\Delta \Phi) / S_{z}, \operatorname{Re}(\Delta \Phi)$ being the phase angle difference between two consecutive straps of the TWA sections.

- For a given strap geometry and applied voltage amplitude to it, the coupling to the plasma of the TWA array scales as the ratio of the total strap number to their inter-distance $n_{\text {str }} / S_{z}$.

- The optimization of the TWA power capability for a given strap maximum voltage requires increasing as much as possible the number of TWA sections and of their ratio $n_{\text {str }} / S_{z}$.

- The study also shows the need for the best adjustment of the other geometrical parameters acting on the coupling and maximum voltage as the length and width of the strap and the depth of the antenna box.

Some of the requirements to obtain the maximum power capability are contradictory as to have large strap width and also ratio $n_{\mathrm{str}} / S_{z}$ they must also accommodate the machine constraints for their implementation. A proposal for the implementation in DEMO is shown in figure 33 where 18 TWA sections are inserted between blanket modules all around the machine. A quantification of the maximum ICRF power capability requires the work of parameter optimization that will only be possible when the space availability, the heating or current drive scenario(s) and a reference plasma profile are defined.

\section{Acknowledgments}

This work has been carried out within the framework of the EUROfusion Consortium and has received funding from the Euratom research and training programme 2014-2018 under grant agreement No. 633053. The views and opinions expressed herein do not necessarily reflect those of the European Commission.

\section{References}

[1] Stix T.H. 1975 Nucl. Fusion 15737

[2] Kazakov Ye.O. et al 2015 Nucl. Fusion 55032001

[3] Kazakov Ye.O. et al 2015 Phys. Plasmas 22082511

[4] Messiaen A. et al 2011 Radio frequency power in plasmas AIP Conf. Proc. 140689

[5] Carpentier S. and Pitts R.A. 2010 Report ITER_D_33y59M_v2,3

[6] Messiaen A. and Weynants R. 2011 Plasma Phys. Control. Fusion $\mathbf{5 3} 085020$

[7] Messiaen A. et al 1984 Proc. 4th Int. Symp. on Heating in Toroidal Plasmas (Roma 21-28 March) ed H. Knoepfel and E. Sindoni (Int. School of Plasma Physics and ENEA) p 315

[8] Messiaen A. et al 2010 Nucl. Fusion 50025026

[9] Lancelotti V. et al 2006 Nucl. Fusion 46 S476

[10] Brambilla M. 1989 Plasma Phys. Control. Fusion 31723

[11] Messiaen A. et al 2009 Nucl. Fusion 49055004

[12] Durodie F. et al 2014 Phys. Plasmas 21061512

[13] Montgomery C.G. et al 1948 Principle of Microwave Circuits (MIT Radiation Laboratory Series vol 8) (New York: McGraw-Hill)

[14] Collins R.E. 1992 Foundations for Microwave Circuits 2nd edn (New York: McGraw-Hill)

[15] Moeller C.P. et al 1992 Radiofrequency heating and current drive Europhysics Conf. Abstract (Brussels, 7-10 July 1992) vol 16E, p 53

[16] Vdovin V.L. 1998 Contributed Papers of 25th EPS Conf. on Controlled Fusion and Plasma Physics Europhysics Conf. Abstract (Praha) vol 22C, p 1438

[17] Ogawa T. et al 2001 Nucl. Fusion 411767

[18] Prater R. et al 2014 Nucl. Fusion 54083024

[19] Bosia G. 2015 Fusion Eng. Design 928

[20] Bosia G. and Ragona R. 2015 Radio frequency power in plasmas AIP Conf. Proc. 1689070001 